\title{
What is the Mass of a Gap-opening Planet?
}

\author{
Ruobing Dong (董若冰 $)^{1}$ and Jeffrey Fung $(\text { 馮澤之 })^{2,3}$ \\ ${ }^{1}$ Steward Observatory, University of Arizona, Tucson, AZ, USA; rdong@email.arizona.edu \\ ${ }^{2}$ Department of Astronomy, University of California, Berkeley, CA, USA \\ Received 2016 November 3; revised 2016 December 12; accepted 2016 December 13; published 2017 January 24
}

\begin{abstract}
High-contrast imaging instruments such as GPI and SPHERE are discovering gap structures in protoplanetary disks at an ever faster pace. Some of these gaps may be opened by planets forming in the disks. In order to constrain planet formation models using disk observations, it is crucial to find a robust way to quantitatively back out the properties of the gap-opening planets, in particular their masses, from the observed gap properties, such as their depths and widths. Combining 2D and 3D hydrodynamics simulations with 3D radiative transfer simulations, we investigate the morphology of planet-opened gaps in near-infrared scattered-light images. Quantitatively, we obtain correlations that directly link intrinsic gap depths and widths in the gas surface density to observed depths and widths in images of disks at modest inclinations under finite angular resolution. Subsequently, the properties of the surface density gaps enable us to derive the disk scale height at the location of the gap $h$, and to constrain the quantity $M_{\mathrm{p}}^{2} / \alpha$, where $M_{\mathrm{p}}$ is the mass of the gap-opening planet and $\alpha$ characterizes the viscosity in the gap. As examples, we examine the gaps recently imaged by VLT/SPHERE, Gemini/GPI, and Subaru/HiCIAO in HD 97048, TW Hya, HD 169142, LkCa 15, and RX J1615.3-3255. Scale heights of the disks and possible masses of the gap-opening planets are derived assuming each gap is opened by a single planet. Assuming $\alpha=10^{-3}$, the derived planet masses in all cases are roughly between 0.1 and $1 M_{\mathrm{J}}$.
\end{abstract}

Key words: circumstellar matter - planet-disk interactions - planets and satellites: formation - protoplanetary disks - stars: pre-main sequence - stars: variables: T Tauri, Herbig Ae/Be

\section{Introduction}

Over the past few years, high angular resolution observations of protoplanetary disks have revolutionized our understanding of planet formation. Rich structures have been identified in disk images in the near-infrared (NIR) and in millimeter/centimeter dust continuum and gas emission. Among them, a particularly intriguing class of features is narrow gaps, found in systems such as TW Hya (Debes et al. 2013; Akiyama et al. 2015; Rapson et al. 2015b; Andrews et al. 2016), HD 169142 (Quanz et al. 2013; Momose et al. 2015), HD 97048 (Ginski et al. 2016; Walsh et al. 2016; van der Plas et al. 2017), AB Aur (Hashimoto et al. 2011), V 4046 Sgr (Rapson et al. 2015a), HD 141569 (Weinberger et al. 1999; Mouillet et al. 2001; Konishi et al. 2016; Perrot et al. 2016), RX J1615.3-3255 (de Boer et al. 2016), and HL Tau (ALMA Partnership et al. 2015; Carrasco-González et al. 2016; Yen et al. 2016). These gaps have both their inner and outer edges revealed in images, enabling a full view of the gap structure, and in particular, a measurement of the gap width. Numerical studies of observational signatures of planet-induced structures have suggested that gaps may be opened by planets (e.g., Wolf \& D'Angelo 2005; Fouchet et al. 2010; Gonzalez et al. 2012; Jang-Condell \& Turner 2012, 2013; Ruge et al. 2013; Dong et al. 2015, 2016; Pinilla et al. 2015a, 2015b; Jin et al. 2016). While giant gaps, found in systems such as transitional disks (e.g., Hashimoto et al. 2012; Follette et al. 2015; Stolker et al. 2016a), may be common gaps opened by multiple planets (Dodson-Robinson \& Salyk 2011; Zhu et al. 2011; Duffell \& Dong 2015), the abovementioned narrow gaps are more likely to be the products of single planets.

\footnotetext{
${ }^{3}$ NASA Sagan Fellow.
}

The masses and locations of planets still forming in disks are key quantities in the study of planet formation. Different formation scenarios, such as "core accretion" and "gravitational instability" models, predict that planets form at different locations, with different final masses. Measuring the masses of gap-opening planets $\left(M_{\mathrm{p}}\right)$ will thus help us distinguish among different planet formation scenarios. To do this, quantitative connections between $M_{\mathrm{p}}$ and gap properties that are directly measurable from images must be established. At the moment, a general scheme for this purpose is lacking. Rosotti et al. (2016) is a pioneer in this direction by connecting $M_{\mathrm{p}}$ with gap width at both $\mathrm{IR}$ and millimeter $(\mathrm{mm})$ wavelengths. They explored a limited parameter space (e.g., no variation in disk viscosity), and it is unclear whether synthetic observations based on the 2D hydro simulations employed by Rosotti et al. (2016) generate the same results as those based 3D hydro simulations.

Our goal is to establish direct and quantitative relations between two observables - the gap depth $\left(\delta_{I}\right.$; the contrast) and width $\left(w_{i}\right.$; the radial extent) - and three fundamental planet and disk parameters $-M_{\mathrm{p}}$, the disk aspect ratio $h / r$, and the $\alpha$-viscosity (Shakura \& Sunyaev 1973). This effort is newly motivated by recent advances in NIR polarized scattered-light imaging driven by Subaru/HiCIAO (Tamura et al. 2006), Gemini/GPI (Macintosh et al. 2008), VLT/NACO (Lenzen et al. 2003), and SPHERE(Beuzit et al. 2008). Currently, these high-resolution imaging instruments are discovering gaps at an ever faster pace, which urgently necessitates the effort presented here.

This $\left[\delta_{I}, w_{I}\right]-\left[M_{\mathrm{p}}, h / r, \alpha\right]$ connection can be segmented into two parts:

1. $\left[\delta_{I}, w_{I}\right]-\left[\delta_{\Sigma}, w_{\Sigma}\right]$ : connect gap depth and width in images to the physical gap depth and width in the gas 
distribution, $\delta_{\Sigma}$ and $w_{\Sigma}$, measured from the disk surface density $\Sigma$, and

2. $\left[\delta_{\Sigma}, w_{\Sigma}\right]-\left[M_{\mathrm{p}}, h / r, \alpha\right]:$ connect $\delta_{\Sigma}$ and $w_{\Sigma}$ to $M_{\mathrm{p}}, h / r$, and $\alpha$.

The second part has been well studied both analytically and numerically. Analytically, Kanagawa et al. (2015, see also Fung et al. 2014; Duffell 2015) have shown that based on torque balancing one can derive a simple scaling relation

$$
\delta_{\Sigma}-1=\frac{\Sigma_{0}}{\Sigma_{\min }}-1 \propto \frac{q^{2}(h / r)^{5}}{\alpha^{2}},
$$

where $\Sigma_{\min }$ and $\Sigma_{0}$ are the depleted and initial (undepleted) surface density in the gap and $q=M_{\mathrm{p}} / M_{\star}$. For the gap width, Goodman \& Rafikov (2001) calculated the propagation of planet-induced density waves, and showed that in the weakly nonlinear regime the waves dissipate and deposit angular momentum over the length scale "shocking length," which is proportional to the disk scale height. It is therefore likely that the width of the gap will also be proportional to the scale height:

$$
w_{\Sigma}=r_{\text {out }}-r_{\text {in }} \propto h,
$$

where $r_{\text {out }}$ and $r_{\text {in }}$ are the radius of the outer and inner gap edges, respectively. This relation will be tested and confirmed with our models. The weakly nonlinear regime applies to planets with masses comparable to or smaller than the thermal mass $M_{\text {thermal }}$,

$$
M_{\mathrm{p}} \lesssim M_{\text {thermal }}=M_{\star}\left(\frac{h}{r}\right)^{3} .
$$

Several groups have carried out numerical simulations to fit $\delta_{\Sigma}$ and $w_{\Sigma}$ as power laws of $M_{\mathrm{p}}, h / r$, and $\alpha$ (Duffell \& MacFadyen 2013; Fung et al. 2014; Duffell 2015; Kanagawa et al. 2015, 2016a). These empirical correlations were synthesized from 2D hydro simulations, and Fung \& Chiang (2016) confirmed that gap opening in 3D simulations produces the same gap profiles as in 2D simulations. The exact functional forms vary somewhat in the literature, mostly due to variations in the definitions of $w_{\Sigma}$ and $\delta_{\Sigma}$. For the gap depth, Fung et al. (2014) found, for deep gaps with $\delta_{\Sigma}$ exceeding 10 , up to $10^{4}$, Equation (1) agrees with simulations to within a factor of 2. For the gap width, hydro simulations by Muto et al. (2010), Dong et al. (2011a, 2011b), Zhu et al. (2013), and Duffell \& MacFadyen (2013) have verified the results of Goodman \& Rafikov (2001), and showed that gap opening indeed initially occurs at a fixed number of scale heights away from the planet's orbit for a given planet mass. By defining the gap width as the radial distance between the two edges where the surface density drops to $50 \%$ of the initial value, Kanagawa et al. (2016a) found $w_{\Sigma} \propto q^{1 / 2}(h / r)^{-3 / 4} \alpha^{-1 / 4}$, which not only has dependencies on $M_{\mathrm{p}}$ and $\alpha$, but also predicts an inverse relation with $h / r$. Whether these differences reflect some unknown physics of gap opening or are simply due to the different definitions of $w_{\Sigma}$ is unclear.

The first step, finding out how a density gap looks like in scattered-light images, is the aim of this paper. We address this question by carrying out $2 \mathrm{D}$ and $3 \mathrm{D}$ hydro and $3 \mathrm{D}$ radiative transfer simulations to study synthetic images of planet-opened gaps with parameterized $h / r$ and $\alpha$. This step is affected by properties of the disk and observational conditions, in particular the angular resolution $\eta$ (a known parameter for a specific observation) and the inclination of the system $i$. The dependence $i$ can be eliminated by only measuring the scattered-light radial profile at $\sim 90^{\circ}$ scattering angle (Section 3.5), such as in face-on disks and along the major axis of inclined disks. A few other factors, including the total disk mass $M_{\text {disk }}$, wavelength $\lambda$, and grain properties, may affect the conversion as well; however, within reasonable ranges in the parameter space assumed in this paper (optically thick, gravitationally stable disks; $0.5 \mu \mathrm{m} \leqslant \lambda \leqslant 2 \mu \mathrm{m}$; close to $90^{\circ}$ scattering angle), they are generally unimportant (e.g., Dong et al. 2012). For extremely faint gaps in which the gap bottom reaches the noise level in the images, the sensitivity of the observations (i.e., detection limit) will affect the measurement of $\delta_{I}$; in this work we focus on relatively low mass planets and shallow gaps and do not consider such a situation.

We focus on narrow gaps $\left(\Delta_{\text {gap }}<0.5 r_{\mathrm{p}}\right)$ opened by a single relatively low mass giant planet about Neptune $\left(M_{\mathrm{N}}\right)$ and Saturn $\left(M_{\mathrm{S}}\right)$ mass, and up to one Jupiter $\left(M_{\mathrm{J}}\right)$ mass. The motivation is mainly threefold. First, such planets are probably more common than their more massive siblings (Cumming et al. 2008; Brandt et al. 2014), and therefore it may be more likely to see gaps opened by such planets. Bowler (2016) concluded that only $\sim 0.6 \%$ of $0.1-3 M_{\odot}$ stars have $5-13 M_{\mathrm{J}}$ planets at orbital distances of $30-300 \mathrm{au}$. Although little is known about the statistics of sub-Jovian planet at tens of astronomical units, observations such as those of HL Tau hint at their existence (Jin et al. 2016). Second, massive planets $\left(\gtrsim M_{\mathrm{J}}\right)$ may open eccentric gaps (e.g., D’Angelo et al. 2006; Kley \& Dirksen 2006), and vortices excited by the Rossby wave instability may form at the gap edge (e.g., Li et al. 2000; Lin \& Papaloizou 2010; Zhu et al. 2014). These features complicate the interpretation of gaps in observations. Third, wide gaps may be common gaps opened by multiple planets, in which case there is a degeneracy between the number of planets and their masses (Dong \& Dawson 2016), preventing unique solutions on the masses of planets. The lower limit of $M_{\mathrm{p}}$ in our work is set so that its gap may be visible in scattered light (e.g., Rosotti et al. 2016).

\section{Simulation Setup}

In this section, we briefly introduce the numerical methods employed to produce synthetic images of gaps. In short, we use the hydrodynamics code PEnGUIn (Fung 2015) to calculate the density structures of planet-opened gaps in $2 \mathrm{D}$ and $3 \mathrm{D}$, then "translate" the resulting density structures to scattered-light images using radiative transfer simulations by the Whitney et al. (2013) Monte Carlo code. For hydro simulations, we follow the procedures described in Fung et al. (2014), while for radiative transfer simulations, we adopt the methods in Dong et al. $(2015,2016)$. Below we briefly summarize the key processes.

\subsection{Hydrodynamical Simulations}

The 2D simulations performed in this paper have an identical setup to the one used in Fung et al. (2014), and the 3D one is identical to Fung \& Chiang (2016). The only differences are that we choose the parameters $q, h / r$, and $\alpha$ from a different parameter space, focusing more on smaller planets and lower disk viscosities, and that all results here are obtained after 6000 orbits, equivalent to about $1 \mathrm{Myr}$ at $30 \mathrm{au}$ around a solar-mass 
Table 1

Model Parameters and Gap Properties in Surface Density and in Face-on Images

\begin{tabular}{|c|c|c|c|c|c|c|c|c|c|c|c|c|}
\hline Model & $q$ & $h / r$ & $\alpha$ & $\delta_{\Sigma}$ & $\begin{array}{l}w_{\Sigma} \\
(\mathrm{au})\end{array}$ & $\Delta_{\Sigma}$ & $\delta_{I}(\eta \mid 0)$ & $\begin{array}{l}w_{I}(\eta \mid 0) \\
(\operatorname{arcsec})\end{array}$ & $\Delta_{I}(\eta \mid 0)$ & $\delta_{I}\left(\eta \mid 0 .{ }^{\prime \prime} 04\right)$ & $\begin{array}{c}w_{I}\left(\eta \mid 0{ }^{\prime \prime} 04\right) \\
\quad(\operatorname{arcsec})\end{array}$ & $\Delta_{I}\left(\eta \mid 0 !^{\prime \prime} 04\right)$ \\
\hline $1 M_{\mathrm{J}} h 5 \alpha 3 \mathrm{e}-4$ & $10^{-3}$ & 0.05 & $3 \times 10^{-4}$ & 803.5 & 11.5 & 0.36 & 49.0 & 0.081 & 0.41 & 31.5 & 0.072 & 0.34 \\
\hline $1 M_{\mathrm{J}} h 5 \alpha 1 \mathrm{e}-3$ & $10^{-3}$ & 0.05 & $10^{-3}$ & 761.4 & 9.7 & 0.32 & 59.5 & 0.068 & 0.33 & 26.2 & 0.064 & 0.30 \\
\hline $1 M_{\mathrm{J}} h 5 \alpha 3 \mathrm{e}-3$ & $10^{-3}$ & 0.05 & $3 \times 10^{-3}$ & 74.9 & 9.8 & 0.32 & 24.2 & 0.066 & 0.31 & 13.9 & 0.063 & 0.30 \\
\hline $1 M_{\mathrm{J}} h 7 \alpha 3 \mathrm{e}-4$ & $10^{-3}$ & 0.07 & $3 \times 10^{-4}$ & 107.1 & 11.8 & 0.38 & 23.7 & 0.077 & 0.35 & 17.6 & 0.071 & 0.33 \\
\hline $1 M_{\mathrm{J}} h 7 \alpha 1 \mathrm{e}-3$ & $10^{-3}$ & 0.07 & $10^{-3}$ & 25.7 & 11.8 & 0.39 & 12.1 & 0.074 & 0.35 & 8.6 & 0.071 & 0.34 \\
\hline $1 M_{\mathrm{J}} h 7 \alpha 3 \mathrm{e}-3$ & $10^{-3}$ & 0.07 & $3 \times 10^{-3}$ & 7.6 & 12.0 & 0.39 & 4.6 & 0.076 & 0.36 & 4.0 & 0.075 & 0.36 \\
\hline $1 M_{\mathrm{S}} h 4 \alpha 3 \mathrm{e}-4$ & $2.9 \times 10^{-4}$ & 0.04 & $3 \times 10^{-4}$ & 78.0 & 8.0 & 0.25 & 30.6 & 0.056 & 0.28 & 12.9 & 0.056 & 0.27 \\
\hline $1 M_{\mathrm{S}} h 4 \alpha 1 \mathrm{e}-3$ & $2.9 \times 10^{-4}$ & 0.04 & $10^{-3}$ & 42.2 & 7.4 & 0.24 & 18.6 & 0.049 & 0.23 & 8.2 & 0.056 & 0.26 \\
\hline $1 M_{\mathrm{S}} h 4 \alpha 3 \mathrm{e}-3$ & $2.9 \times 10^{-4}$ & 0.04 & $3 \times 10^{-3}$ & 9.9 & 7.7 & 0.25 & 6.4 & 0.052 & 0.25 & 4.2 & 0.060 & 0.28 \\
\hline $1 M_{\mathrm{S}} h 5 \alpha 3 \mathrm{e}-4$ & $2.9 \times 10^{-4}$ & 0.05 & $3 \times 10^{-4}$ & 30.9 & 8.4 & 0.28 & 17.7 & 0.056 & 0.28 & 8.6 & 0.057 & 0.27 \\
\hline $1 M_{\mathrm{S}} h 5 \alpha 1 \mathrm{e}-3$ & $2.9 \times 10^{-4}$ & 0.05 & $10^{-3}$ & 11.2 & 8.5 & 0.28 & 6.8 & 0.057 & 0.27 & 4.7 & 0.060 & 0.29 \\
\hline $1 M_{\mathrm{S}} h 5 \alpha 3 \mathrm{e}-3$ & $2.9 \times 10^{-4}$ & 0.05 & $3 \times 10^{-3}$ & 4.1 & 8.8 & 0.29 & 3.4 & 0.057 & 0.27 & 2.6 & 0.063 & 0.29 \\
\hline $1 M_{\mathrm{S}} h 7 \alpha 3 \mathrm{e}-4$ & $2.9 \times 10^{-4}$ & 0.07 & $3 \times 10^{-4}$ & 6.3 & 11.1 & 0.36 & 4.3 & 0.073 & 0.34 & 3.7 & 0.073 & 0.35 \\
\hline $1 M_{\mathrm{S}} h 7 \alpha 1 \mathrm{e}-3$ & $2.9 \times 10^{-4}$ & 0.07 & $10^{-3}$ & 3.1 & 11.6 & 0.38 & 2.3 & 0.071 & 0.34 & 2.2 & 0.075 & 0.36 \\
\hline $1 M_{\mathrm{S}} h 7 \alpha 3 \mathrm{e}-3$ & $2.9 \times 10^{-4}$ & 0.07 & $3 \times 10^{-3}$ & 1.7 & 11.8 & 0.37 & 1.6 & 0.071 & 0.34 & 1.4 & 0.081 & 0.37 \\
\hline $2 M_{\mathrm{N}} h 4 \alpha 3 \mathrm{e}-4$ & $10^{-4}$ & 0.04 & $3 \times 10^{-4}$ & 10.6 & 6.3 & 0.21 & 7.8 & 0.045 & 0.22 & 4.1 & 0.052 & 0.25 \\
\hline $2 M_{\mathrm{N}} h 4 \alpha 1 \mathrm{e}-3$ & $10^{-4}$ & 0.04 & $10^{-3}$ & 4.7 & 6.6 & 0.22 & 4.1 & 0.045 & 0.21 & 2.7 & 0.057 & 0.27 \\
\hline $2 M_{\mathrm{N}} h 4 \alpha 3 \mathrm{e}-3$ & $10^{-4}$ & 0.04 & $3 \times 10^{-3}$ & 2.4 & 6.9 & 0.22 & 2.2 & 0.045 & 0.21 & 1.8 & 0.061 & 0.28 \\
\hline $2 M_{\mathrm{N}} h 5 \alpha 3 \mathrm{e}-4$ & $10^{-4}$ & 0.05 & $3 \times 10^{-4}$ & 4.5 & 7.7 & 0.26 & 4.0 & 0.055 & 0.26 & 3.0 & 0.059 & 0.28 \\
\hline $2 M_{\mathrm{N}} h 5 \alpha 1 \mathrm{e}-3$ & $10^{-4}$ & 0.05 & $10^{-3}$ & 2.5 & 8.2 & 0.27 & 2.1 & 0.058 & 0.28 & 1.9 & 0.066 & 0.31 \\
\hline $2 M_{\mathrm{N}} h 5 \alpha 3 \mathrm{e}-3$ & $10^{-4}$ & 0.05 & $3 \times 10^{-3}$ & 1.6 & 8.8 & 0.28 & 1.5 & 0.053 & 0.25 & $\mathrm{~N} / \mathrm{A}^{\mathrm{a}}$ & $\mathrm{N} / \mathrm{A}$ & $\mathrm{N} / \mathrm{A}$ \\
\hline $2 M_{\mathrm{N}} h 7 \alpha 3 \mathrm{e}-4$ & $10^{-4}$ & 0.07 & $3 \times 10^{-4}$ & 1.6 & 13.3 & 0.43 & 1.5 & 0.085 & 0.41 & 1.4 & 0.086 & 0.39 \\
\hline $1 M_{\mathrm{N}} h 4 \alpha 3 \mathrm{e}-4$ & $5.1 \times 10^{-5}$ & 0.04 & $3 \times 10^{-4}$ & 3.5 & 6.1 & 0.20 & 3.2 & 0.044 & 0.20 & 2.4 & 0.053 & 0.25 \\
\hline $1 M_{\mathrm{N}} h 4 \alpha 1 \mathrm{e}-3$ & $5.1 \times 10^{-5}$ & 0.04 & $10^{-3}$ & 2.1 & 6.5 & 0.21 & 1.9 & 0.046 & 0.21 & 1.6 & 0.061 & 0.28 \\
\hline $1 M_{\mathrm{N}} h 4 \alpha 3 \mathrm{e}-3$ & $5.1 \times 10^{-5}$ & 0.04 & $3 \times 10^{-3}$ & 1.4 & 7.0 & 0.22 & 1.3 & 0.051 & 0.23 & $\mathrm{~N} / \mathrm{A}$ & $\mathrm{N} / \mathrm{A}$ & $\mathrm{N} / \mathrm{A}$ \\
\hline
\end{tabular}

Notes. See the definition of parameters in Section 3.2.

a Gap undefined by our definitions (Figure 4).

star. This time is sufficient for models with $\alpha \geqslant 10^{-3}$ to reach a quasi-steady state. For the models with the lowest $\alpha$ values, the gaps have not yet fully settled, but they are still relevant models since $1 \mathrm{Myr}$ is already a significant fraction of the typical protoplanetary disk lifetime. We recapitulate some important features here and refer the reader to Fung et al. (2014) and Fung \& Chiang (2016) for the details.

The initial disk profile assumes the following surface density and sound speed profiles:

$$
\begin{aligned}
\Sigma_{0} & =\Sigma_{\mathrm{p}}\left(\frac{r}{r_{\mathrm{p}}}\right)^{-\frac{1}{2}}, \\
c_{\mathrm{s}} & =c_{\mathrm{p}}\left(\frac{r}{r_{\mathrm{p}}}\right)^{-\frac{1}{2}},
\end{aligned}
$$

where we set $\Sigma_{\mathrm{p}}=1,{ }^{4}$ and $c_{\mathrm{p}}$ is a parameter we vary to obtain different $h / r$ values. Since the disk scale height $h$ is $c_{\mathrm{s}} / \Omega_{\mathrm{k}}$, with $\Omega_{\mathrm{k}}$ being the Keplerian frequency, $h / r$ is constant in radius. In $3 \mathrm{D}$ hydrostatic equilibrium, the initial density structure is

$$
\rho_{0}=\rho_{\mathrm{p}}\left(\frac{r}{r_{\mathrm{p}}}\right)^{-\frac{3}{2}} \exp \left(\frac{G M}{c_{\mathrm{s}}^{2}}\left[\frac{1}{\sqrt{r^{2}+z^{2}}}-\frac{1}{r}\right]\right),
$$

where $\rho_{\mathrm{p}}=\Sigma_{\mathrm{p}} / \sqrt{2 \pi h_{\mathrm{p}}^{2}}$, with $h_{\mathrm{p}}$ being the scale height at $r=r_{\mathrm{p}}$, is the initial gas density at the location of the planet. Density and sound speed are related by a locally isothermal

\footnotetext{
4 Since we do not consider the self-gravity of the disk, this normalization has no impact on our results.
}

equation of state. The kinematic viscosity in the disk is $\nu=\alpha c_{\mathrm{s}}$ $h$, where $\alpha$ is constant in radius. The simulation grid spans $0.4 r_{\mathrm{p}}$ to $2 r_{\mathrm{p}}$ in radius, which corresponds to $12-60 \mathrm{au}$, if the planet is placed at $30 \mathrm{au}$. Our planet is fixed on its orbit and disk-driven radial migration is not taken into account; migration may affect the properties of the gap if it is fast (Malik et al. 2015).

Table 1 lists the parameters and results for all models.

\subsection{Monte Carlo Radiative Transfer Simulations}

For radiative transfer calculations, we assume a central source of $2.325 R_{\odot}$ and $4350 \mathrm{~K}$, appropriate for a $1 M_{\odot}$ star at $1 \mathrm{Myr}$ (Baraffe et al. 1998). For 3D hydro simulations, we directly feed the density grid into the radiative transfer simulations; for 2D hydro simulations, we puff up the disk surface density in the vertical direction $z$ by a Gaussian profile:

$$
\rho(z)=\frac{\Sigma}{h \sqrt{2 \pi}} e^{-z^{2} / 2 h^{2}},
$$

using the same scale height $h$ as in the hydro simulations. The Monte Carlo radiative transfer (MCRT) simulation domain spans $\pm 30^{\circ}$ from the disk midplane, which is about a factor of 2 higher than the scattered-light surface. A 1 au-radius circumplanetary region centered on the planet is excised (except in Section 2.3 and Figure 2; see below), for reasons laid out in Section 2.3.

The hydro simulations are gas only, while scattered light comes from the dust. We convert gas into dust by assuming the two are well mixed, and assume interstellar medium grains 
(Kim et al. 1994) for our dust model. These grains are submicron in size, and their scattering properties are calculated using Mie theory. They are small enough that their stopping time is very short compared to their orbital timescale and the vertical stirring timescale (characterized by the viscous parameter $\alpha$ ) in our simulations, so they are dynamically well coupled with the gas (e.g., Zhu et al. 2012).

We scale the size of the hydro grid so that the planet is at $30 \mathrm{au}$. Given the inner working angles (IWA) in current GPI and SPHERE observations $(\sim 0$ !" $1=14$ au at $140 \mathrm{pc}), 30 \mathrm{au}$ is about as close as a planet can be for the inner gap edge to still be outside the IWA. The inner dust disk edge is taken to be the dust sublimation radius $r_{\text {sub }}$, where dust reaches a temperature of $1600 \mathrm{~K}$; between $r_{\text {sub }}$ and the inner boundary of hydro simulations (12 au), we assume an axisymmetric inner disk smoothly joining the outer hydro disk with a constant surface density. The details of the inner disk does not matter as long as it is axisymmetric and does not cast significant shadows on the other disk. We scale the hydro disk so that the initial condition $\Sigma_{0}(r)=10(r / 30 \mathrm{au})^{-1 / 2} \mathrm{~g} \mathrm{~cm}^{-2}$, resulting in a final total dust disk mass between $5 \times 10^{-5}$ to $10^{-4} M_{\odot}$ inside $60 \mathrm{au}$ (depending on how deep the gap is). We note that gap width and contrast do not depend on the assumed disk mass within one order of magnitude from the chosen value. Radiative transfer simulations produce full-resolution polarized intensity (PI) images at $H$-band, which are convolved by a Gaussian point-spread function to achieve the desired angular resolutions. Fiducially we choose $\eta=0$ " 04 , comparable to what is achievable by SPHERE and GPI, unless otherwise noted.

As examples, Figure 1 shows the dust surface density and scattered-light images at both face-on and $i=45^{\circ}$ inclinations for Model $1 M_{\mathrm{S}} h 5 \alpha 1 \mathrm{e}-3$. Unless otherwise noted, all images in this paper show $H$-band PI $I$, although we note that our results generally apply to total intensity images as well (see below). In panels (e) and (f), we scale the images by $r^{2}$ to compensate for the distance from the star, where $r$ is the distance from the star (deprojected in inclined cases). Unless otherwise noted, all $\mathrm{H}$ band images and their radial profiles below have been scaled by $r^{2}$.

\section{3. $2 D$ and 3D Gaps Are the Same in Scattered Light}

Fung \& Chiang (2016) have shown that the surface density of planet-opened gaps in 3D hydro simulations are nearly the same as the gaps in $2 \mathrm{D}$ simulations. In this section we show that synthetic images are also practically identical between a 3D model and a model with a 2D surface density manually puffed up using Equation (7).

Figure 2 shows two face-on synthetic images and their azimuthally averaged radial profile for the model $1 M_{\mathrm{S}} h 5 \alpha 1 \mathrm{e}-3$. Panel (a) is from a 3D hydro simulation, while panel (b) is produced by puffing up the surface density of the model by the same assumed $h / r$ profile as in the 3D hydro calculation. Note that unlike all other images in the paper, in both panels the circumplanetary region is not excised. The gap profiles (c) from the two images are essentially identical. This result means vertical kinematic support is unimportant in the vertical distribution of material in the gap region, and we can safely puff up 2D hydro surface density structures in MCRT simulations to study gaps in scattered light.

The differences between the two images are in the local structures. First, the spiral arms are clearly more prominent in (a) than in (b), due to vertical kinematic support in planet- induced density waves (Zhu et al. 2015; Dong \& Fung 2016). Second, the circumplanetary region sticks out in (b) while it is not noticeable in (a). This is because when puffing up 2D structures into $3 \mathrm{D}$, the circumplanetary region, which has a higher surface density than the surroundings, is artificially expanded in the vertical direction by the same $h / r$ as the rest of the disk. In reality though, the gravity in the circumplanetary region is dominated by the planet so the actual $h / r$ of the circumplanetary region is much smaller. This leads to artificial brightening in scattered light of the region in (b). For this reason, and to avoid the artificial shadow in the outer disk produced by this structure (e.g., Jang-Condell 2009), we excise the circumplanetary region in $2 \mathrm{D}$ surface density maps when puffing it up in MCRT simulations in the rest of the paper.

\section{The Conversions}

In this section, we explore the connections between gap properties in surface density and disk and planet parameters $h / r, \alpha$, and $M_{\mathrm{p}}$, and the correlations between gaps in scattered light and gaps in surface density.

\subsection{The Qualitative Picture}

Figure 3 shows the radial profiles of the surface density, and both full-resolution and convolved images at face-on viewing angle for three representative models with $\alpha=10^{-3}$, $h / r=0.05$, and $M_{\mathrm{p}}=2 M_{\mathrm{N}}, 1 M_{\mathrm{S}}$, and $1 M_{\mathrm{J}}$. The images have been scaled by $r^{2}$. Qualitatively, in all three cases, each with very different gap depths, the radial profiles of the fullresolution images closely traces the variations in the surface density profiles. This general behavior has been found by Muto (2011) using analytical calculations of scattered-light grazing angles in disks with narrow gaps. The two deviate near the gap bottom, where the scattered-light profile does not reach as deep as the surface density profile. Also, the outer edge of the scattered-light gap lies slightly inside the surface density gap.

These observations can be qualitatively explained. Approximately, scattered light comes from a surface with optical depth of unity to the star (Takami et al. 2014). For disks with constant $h / r$, if the surface density variation is smooth, this surface is flat at roughly a constant polar angle $\theta_{\text {surface }} \propto h / r$ (i.e., not concave-up as in flared disks). In this case, at $i=0$ the $r^{2}$ scaled intensity of the scattered light at each radius is roughly proportional to the surface density of the dust above the surface, $I \propto \Sigma_{\theta}>\theta_{\text {surface }}(r)$. Because $\Sigma_{\theta}>\theta_{\text {surface }}(r) \propto \Sigma(r)$ for disks with constant $h / r$, it follows that $I \propto \Sigma(r)$, which is what we find. This relation breaks when the surface density fluctuation is so large that the disk surface is no longer at roughly a constant $\theta_{\text {surface, }}$ which happens when the gap depletion is high and at the gap edges. Also, this analysis does not formally hold if the disk surface is flared (i.e., $h / r$ increases outward). However, gaps are local structures, and the global flareness of the disk may not significantly affect the width and depth of local structures. We will come back to this point later in Section 3.6.

Convolving the full-resolution image by a finite PSF smooths structures. Convolved images (dotted line) thus closely follow full-resolution images outside the gap, as the variations occur on a spatial scale much larger than the angular resolution ( $\sim 6$ au for $\eta=0$ !. 04 and $d=140 \mathrm{pc}$ ). Inside the gaps, convolved images have less steep edges and shallower depths. 

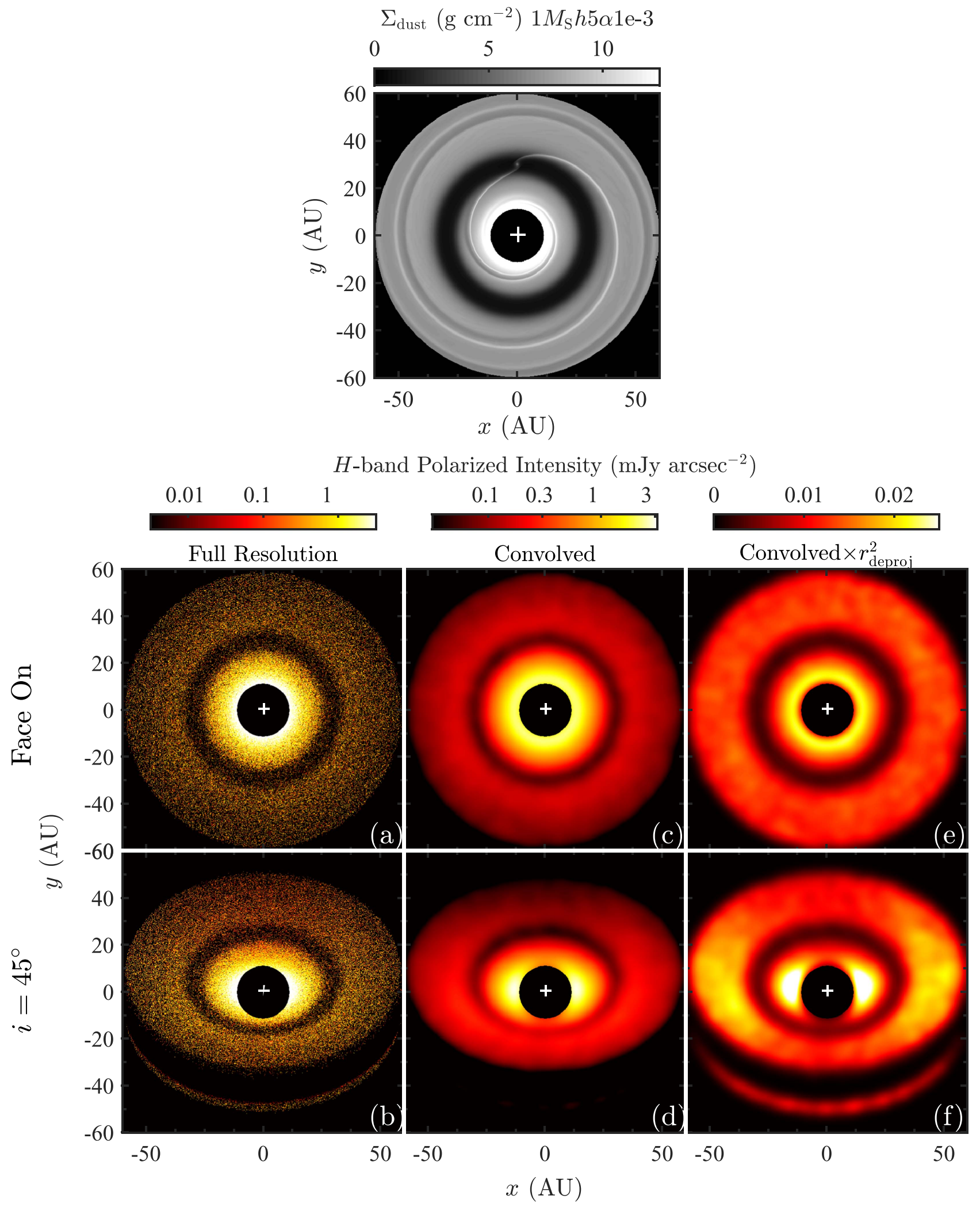

Figure 1. Dust surface density (top) and $H$-band PI images at (a), (c), (d) face-on and (b), (d), (f) $i=45^{\circ}$ inclinations of the $1 M_{\mathrm{S}} h 5 \alpha 1 \mathrm{e}-3$ model. The image panels are (a), (b) full-resolution images, (c), (d) convolved images with $\eta=0$ !' 04 , and (e), (f) convolved images scaled by $r^{2} ; r$ is the deprojected distance from the star. See Section 2.2 for details.

\subsection{The Definitions of Gap Parameters}

A planet-opened gap is a perturbation on top of an initial "background" disk with no planets. The definition and characterization of a gap are relative to this background. While in simulations a planet-less background can be well defined, in real observations of actual systems it is nearly impossible to do so. In addition, a complete global disk profile is often not 
Full Resolution $H$-band PI $\times r^{2}\left(\mathrm{mJy} \operatorname{arcsec}^{-2}\right)$

\begin{tabular}{lll}
0 & 0.05 & 0.1 \\
\hline
\end{tabular}
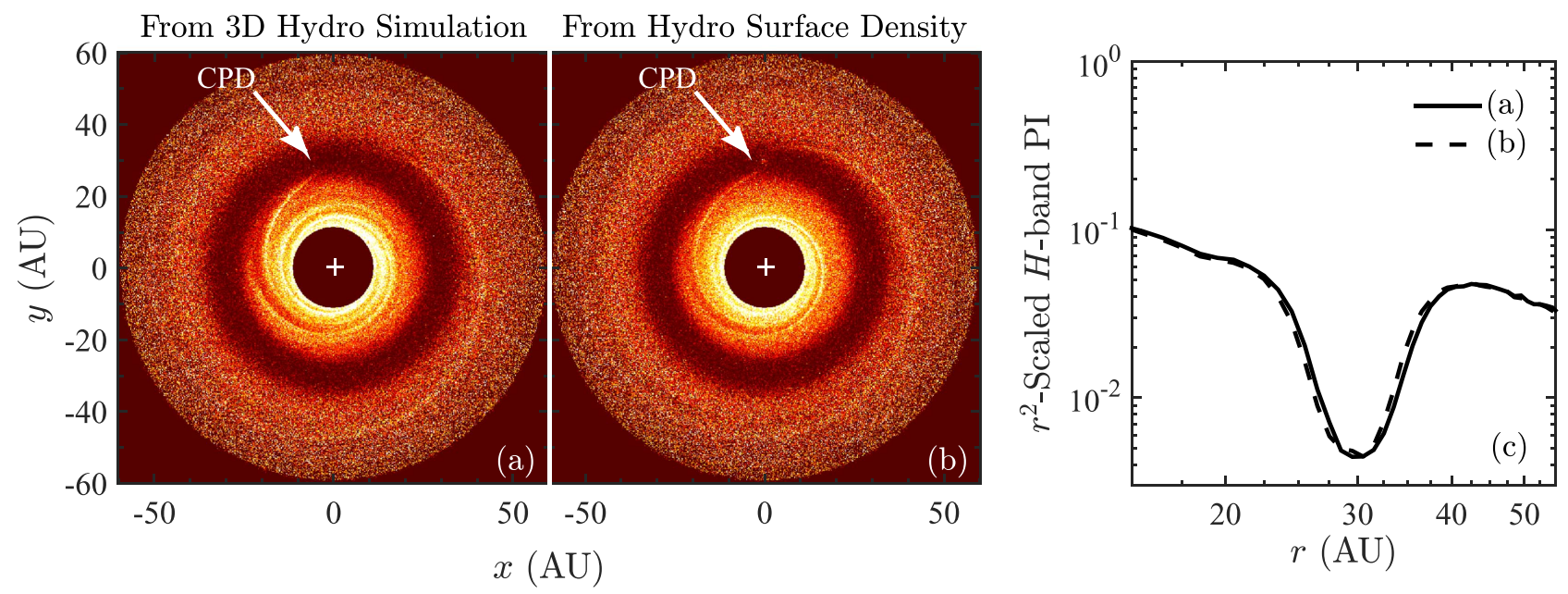

Figure 2. Left: face-on images of a disk with $h / r=0.05, \alpha=10^{-3}$, and a $0.5 M_{\mathrm{J}}$ planet at 30 au. Image (a) is from a 3D hydro simulation, while image (b) is produced by puffing up the surface density of the model by the same $h / r$ profile as in the 3D hydro calculation. Note that in both panels the circumplanetary region (CPD) is not excised (indicated by the arrow). Panel (c): radial profile of the two images. The gap in the two images is essentially identical. See Section 2.3 for details.

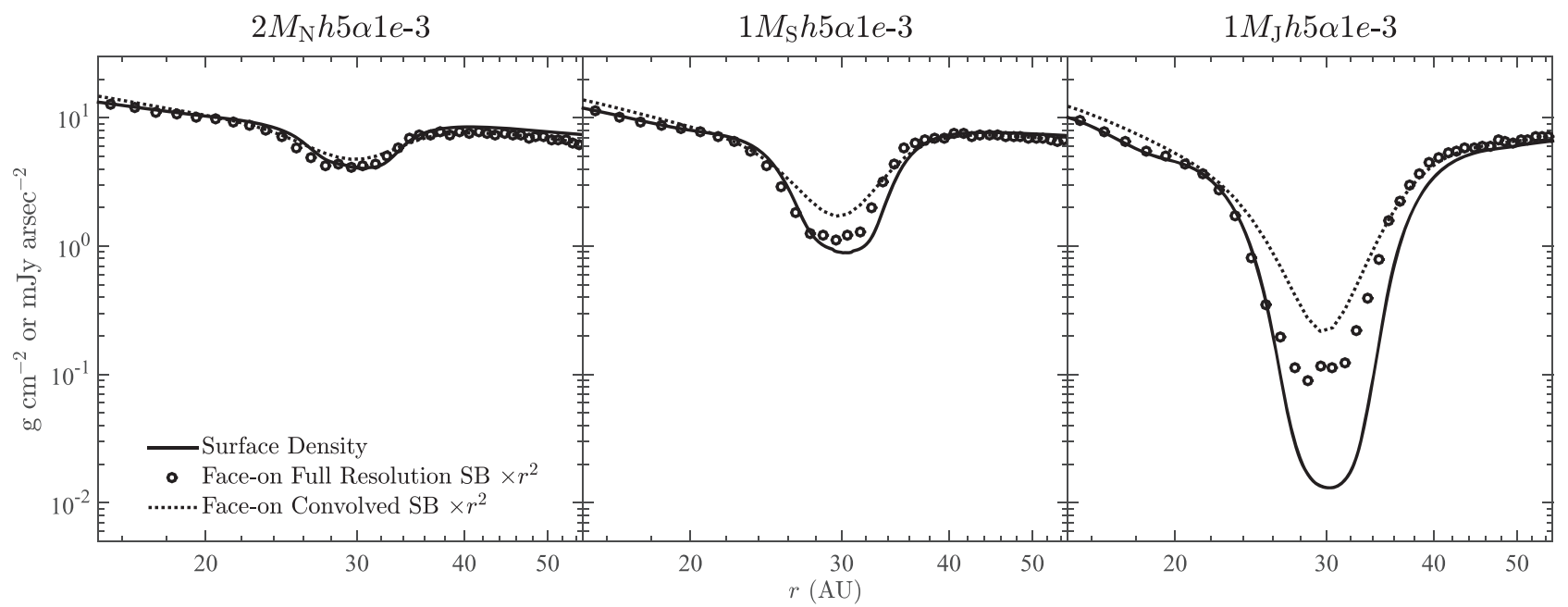

Figure 3. Radial profiles of the surface density (solid) and $H$-band polarized intensity surface brightness (circles: full resolution; dotted line: convolved; both scaled by $r^{2}$ ) of three models with $h / r=0.05, \alpha=10^{-3}$, and $M_{\mathrm{p}}=2 M_{\mathrm{N}}, 1 M_{\mathrm{S}}$, and $1 M_{\mathrm{J}}$, from left to right. Both the full-resolution and convolved surface brightness curves have been scaled by the same constant factor so that they roughly meet the surface density curves at $r=15 \mathrm{au}$.

accessible in real observations with finite inner and outer working angles. In fact, based on scattered light alone it is usually difficult to judge whether a "gap-like" structure is indeed a planet-opened gap or not. To mimic actual observations and to maximize the applicability of the correlations derived in this work, below we will assume gaps as local structures so that its parameterization only involves its immediate neighborhood, and we will characterize an observed gap in such a way that does not require prior knowledge of the underlying planet-less background disk. We emphasize that this is a necessary assumption in order to construct useful empirical relations to quickly and quantitatively convert observed gap properties to planet and disk properties. For individual actual systems, whether this assumption is good or not may be indicated by the reasonableness of the fitting. More accurate assessments of the planet and disk properties require detailed modeling of individual systems with specifically designed simulations to match the 1D (or even 2D) gap profiles, instead of using parameterized gap depth and width.

The definitions of gap depth and width in surface density vary somewhat in the literature. For the former, the common practice is to define it as the ratio between the minimum $\Sigma$ inside the gap (or averaged over a finite radial range) to a fiducial "undepleted" value $\left(\Sigma_{0}\right)$. What counts as $\Sigma_{0}$ varies. For the gap width, the difficulty lies in defining the two gap edges, $r_{\text {out }}$ and $r_{\text {in }}$ (such that $w_{\Sigma}=r_{\text {out }}-r_{\text {in }}$ ). The common practice is to define them as where $\Sigma$ reaches a certain threshold fraction of $\Sigma_{0}$ (e.g., Kanagawa et al. 2016a). This class of width definitions does not work for shallow gaps in which the depletion is less than the threshold fraction. With this fixeddepth-width definition, $w_{\Sigma}$ depends on $M_{\mathrm{p}}$ and $\alpha$ in addition to $h / r$ (e.g., Kanagawa et al. 2016a, 2016b).

In this paper, we adopt dynamical definitions of the gap depth and width in both surface density and images, as illustrated in Figure 4. Our definitions work for gaps with a 

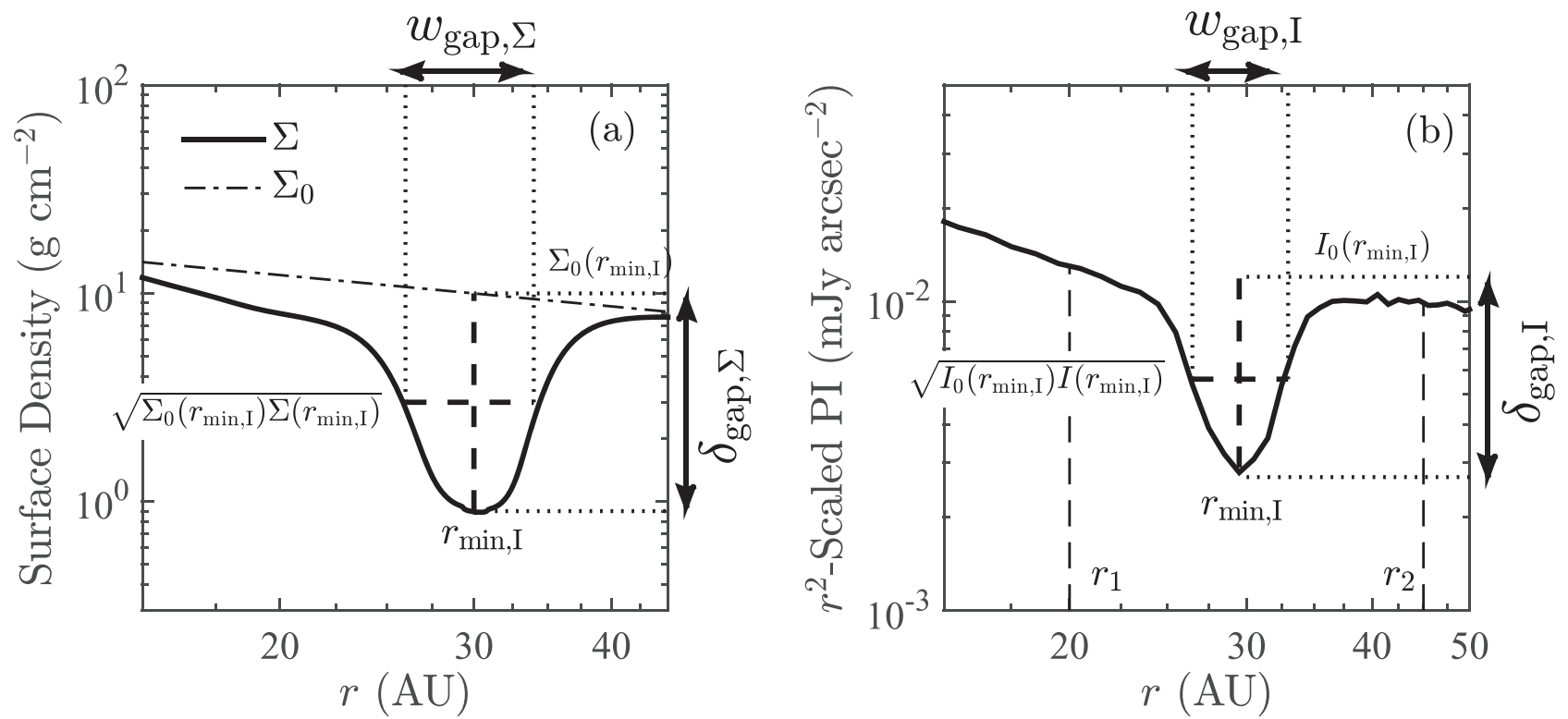

Figure 4. Definitions of (a) $w_{\Sigma}$ and $\delta_{\Sigma}$ in surface density, and (b) $w_{I}$ and $\delta_{I}$ in scattered-light image. The gap depth (the vertical dashed segment) is defined as the ratio between an "undepleted background" and the minimum value of the quantity inside the gap at radius $r_{\min }\left(r_{\min , \Sigma}\right.$ or $r_{\min , \mathrm{I}} ; \approx r_{\mathrm{p}}=30$ au): $\delta_{\Sigma}=\Sigma_{0}\left(r_{\min , \Sigma}\right) / \Sigma\left(r_{\min , \Sigma}\right)$ and

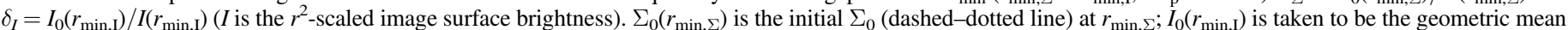
of $I$ at $r_{1}=(2 / 3) r_{\mathrm{min}, \mathrm{I}}$ and $r_{2}=(3 / 2) r_{\mathrm{min}, \mathrm{I}}$ (marked in (b)). $r_{1}$ and $r_{2}$ are far away from the gap, and for narrow gaps, planet-induced perturbation should be small at these distances. The gap width (horizontal dashed segment) is defined as the distance between the inner and outer edges of the gap (two vertical dotted lines), the radius where the quantities reach the geometric mean of the background and the gap minimum (i.e., $\sqrt{\Sigma_{0}\left(r_{\min , \Sigma}\right) \Sigma\left(r_{\min , \Sigma}\right)}$ and $\left.\sqrt{I_{0}\left(r_{\text {min,I }}\right) I\left(r_{\min , \mathrm{I}}\right)}\right)$.

wide range of depth and width. For $\Sigma$, we first find the location in the gap where $\Sigma$ reaches the minimum, $r_{\min , \Sigma}$. The initial value of $\Sigma$ at $r_{\min , \Sigma}, \Sigma_{0}\left(r_{\min }\right)$, is regarded as the fiducial undepleted value. Thus $\delta_{\Sigma}$ is defined as

$$
\delta_{\Sigma} \equiv \frac{\Sigma_{0}\left(r_{\min , \Sigma}\right)}{\Sigma\left(r_{\min , \Sigma}\right)} .
$$

We then regard the inner and outer gap edges, $r_{\mathrm{in}, \Sigma}$ and $r_{\mathrm{out}, \Sigma}$, as where the surface density reaches the geometric mean of the minimum and the undepleted value,

$$
\begin{aligned}
\Sigma_{\text {edge }} & =\Sigma\left(r_{\text {in }, \Sigma}\right)=\Sigma\left(r_{\text {out }, \Sigma}\right) \\
& =\sqrt{\Sigma_{0}\left(r_{\min , \Sigma}\right) \Sigma\left(r_{\min , \Sigma}\right)} ;
\end{aligned}
$$

therefore, the gap width $w_{\Sigma}$ is

$$
w_{\Sigma} \equiv r_{\text {in }, \Sigma}-r_{\text {out }, \Sigma},
$$

and the normalized gap width $\Delta_{\Sigma}$ is defined as

$$
\Delta_{\Sigma} \equiv \frac{w_{\Sigma}}{r_{\min , \Sigma}}
$$

We note that $w_{\Sigma}$ has a physical unit (e.g., au or arcsec), while $\Delta_{\Sigma}$ is dimensionless.

The definitions of gap width and gaps in scattered light are similar. We first scale the image by $r^{2}$, then find the location in the gap where $I$ reaches the minimum, $r_{\text {min,I }}$ (a gap cannot be defined if the $r^{2}$-scaled intensity monotonically varies with radius). We find that in general $r_{\text {min,I }} \sim r_{\min , \Sigma} \sim r_{\mathrm{p}}$, with small model-by-model differences. We then read the intensity at $r_{1}=2 r_{\text {min,I }} / 3$ and $r_{2}=3 r_{\text {min, I }} / 2$. These two locations are far away from the gap region so they are considered to be outside the influence of the gap. A fiducial "background" $I_{0}$ at $r_{\min }$ is subsequently defined as the geometric mean of the two points (note that this choice assumes the underlying "planet-less" background profile follows a power law),
$I_{0}\left(r_{\min , \mathrm{I}}\right) \equiv \sqrt{I\left(r_{1}\right) I\left(r_{2}\right)}$, and the gap depth is

$$
\delta_{I} \equiv I_{0}\left(r_{\min , \mathrm{I}}\right) / I\left(r_{\min , \mathrm{I}}\right) .
$$

The inner and outer edges of the gap, $r_{\mathrm{in}, \mathrm{I}}$ and $r_{\mathrm{in}, \mathrm{I}}$, are found where $I$ reaches the geometric mean of $I_{0}$ and $I$ at $r_{\text {min,I }}$ :

$$
I_{\text {edge }}=I\left(r_{\text {in }, \mathrm{I}}\right)=I\left(r_{\text {out }, \mathrm{I}}\right)=\sqrt{I_{0}\left(r_{\min , \mathrm{I}}\right) I\left(r_{\min , \mathrm{I}}\right)} .
$$

Correspondingly, the width of the gap $w_{I}$ is defined as

$$
w_{I} \equiv r_{\mathrm{in}, \mathrm{I}}-r_{\mathrm{out}, \mathrm{I}},
$$

and the normalized gap width $\Delta_{I}$ is

$$
\Delta_{I} \equiv \frac{w_{I}}{r_{\min , I}}
$$

The gap parameters in the surface density and face-on images for all models are listed in Table 1.

Finally, we note that all image-related quantities, such as $\delta_{I}$, $w_{I}$, and $\Delta_{I}$, are functions of both angular resolution $\eta$ and inclination $i$, i.e., $\delta_{I}(\eta, i), w_{I}(\eta, i)$, and $\Delta_{I}(\eta, i)$. We omit the variables when the context is clear.

\subsection{The Correlations between $\left[\alpha, h / r, M_{p}\right]$ and $\left[\Delta_{\Sigma}, \delta_{\Sigma}\right]$}

Previous numerical studies have fit empirical relations to express $w_{\Sigma}$ and $\delta_{\Sigma}$ as functions of $\left[\alpha, h / r, M_{\mathrm{p}}\right]$. Here we derive our version based on the new definitions of depth and width in Figure 4. As discussed in Section 1, analytical weakly nonlinear theory and simple scaling relations based on torque balancing have motivated Equations (1) and (2) as possible functional forms for $w_{\Sigma}$ and $\delta_{\Sigma}$. We are able to achieve a good fit for both correlations, as shown in Figure 5, by varying only the constant proportionality factor. For gap width we have

$$
\Delta_{\Sigma}=5.8 \frac{h}{r}
$$


Eqn. (13)

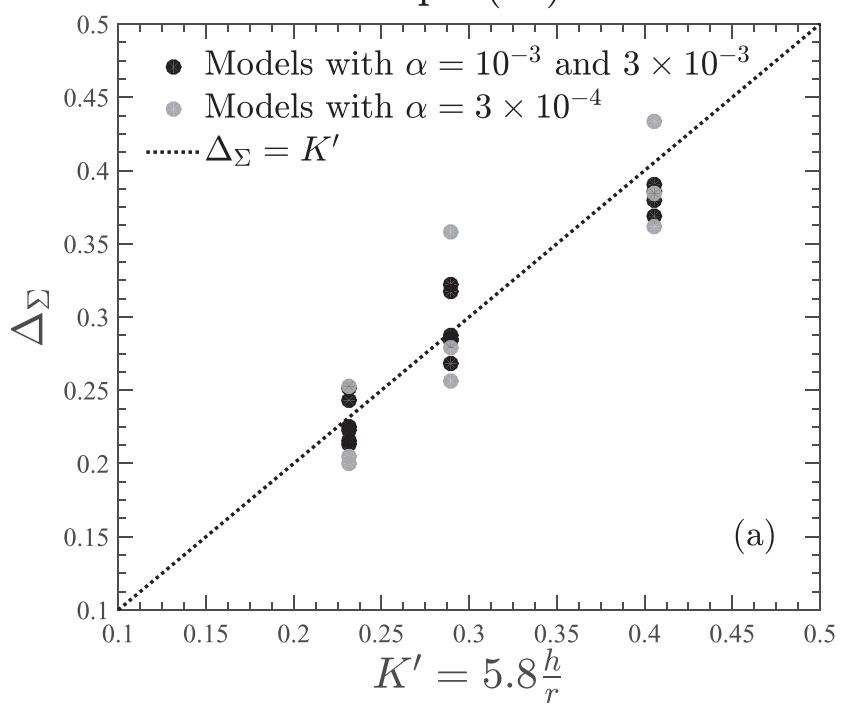

Eqn. (14)

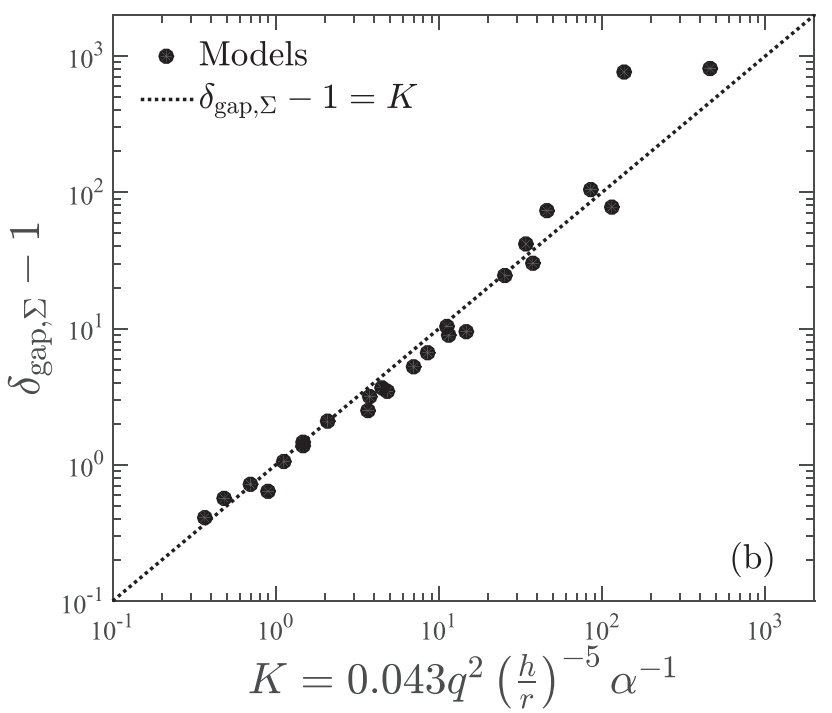

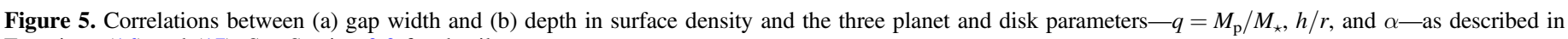
Equations (16) and (17). See Section 3.3 for details.

The standard deviation $\sigma$ is $9 \%$ for all models. If we restrict to only the models that have been run for longer than viscous timescale $\left(\alpha=10^{-3}\right.$ and $\alpha=3 \times 10^{-3}$, black points), we again recover Equation (16) while $\sigma$ shrinks to $6 \%$. We note that if we adopt the Kanagawa et al. (2016a) definition of the gap width we do recover their $\Delta_{\Sigma}=0.41 q^{1 / 2}(h / r)^{-3 / 4} \alpha^{-1 / 4}$ correlation (with a standard deviation of $17 \%$ ). However, we prefer our dynamical definition of the gap width over defining the width as the distance between two points on the edges with a fixed depletion factor for a few reasons. First, the latter does not work for shallow gaps opened by relatively low mass planets, and in real observations such gaps are more common than deeper gaps opened by more massive planets, due to the higher occurrence rate of smaller planets than their more massive siblings (Cumming et al. 2008). Second, adopting the latter definition returns a less robust correlation between the gap width in images and the gap width in surface density (not shown). Third, while the Kanagawa et al. (2016a) correlation is a pure empirical correlation synthesized from simulations within a confined parameter space, our Equation (16) has some theoretical footing from gap-opening theory, and thus is more robust when applied to real systems that may lie outside the parameter space explored by our models.

For gap depth, we have

$$
\delta_{\Sigma}-1=0.043 q^{2}\left(\frac{h}{r}\right)^{-5} \alpha^{-1},
$$

with $\sigma=17 \%$ for all but the three $M_{\mathrm{p}}=M_{\mathrm{J}}$ and $h / r=0.05$ models, which have the largest thermal masses in our models, $M_{\mathrm{p}}=8 M_{\text {thermal }}$, and so their gap-opening process is in the strongly nonlinear regime and the analytical torque balancing calculations break. We have experimented with freeing the power-law indexes in the fit; overall, no significant improvement is found.

We thus conclude that the gap width and depth definitions in Figure 4 successfully capture the physics of gap opening as argued by analytical theories. One thing to note is that $h / r$ can now be directly constrained based on the measured gap properties. Assuming hydrostatic equilibrium in the vertical direction, the scale height $h / r$ is related to the disk temperature $T$ at each radius as

$$
\frac{h}{r}=\frac{c_{\mathrm{s}}}{v_{\mathrm{k}}}=\frac{\sqrt{k T} / \mu}{v_{\mathrm{k}}},
$$

where $v_{\mathrm{k}}$ is the Keplerian speed and $\mu$ is the mean molecular weight. Thus, our constraints on $h / r$ based on the measured gap properties can be used to infer the midplane temperature of the disk at the location of the gap.

\subsection{The Correlations between Gap Properties in Surface Density and in Face-on Images}

At $i=0$, we measure four quantities for each model for a given $\eta$ : $\Delta_{\Sigma}, \delta_{\Sigma}, \Delta_{I}(\eta)$, and $\delta_{I}(\eta)$. Figure 6 compares $\Delta_{\Sigma}$ with $\Delta_{I}(\eta)$, and $\delta_{\Sigma}$ with $\delta_{I}(\eta)$, for both $\eta=0$ and $\eta=0$ "' 04 .

In full-resolution images, it is well approximated that $\Delta_{I}(0)=\Delta_{\Sigma}$, with a standard deviation of $7 \%$ for all gaps. For the depth, $\delta_{I}(0)$ is close to $\delta_{\Sigma}$ for shallow gaps $\left(\delta_{\Sigma} \lesssim 5\right)$, but deviates from $\delta_{\Sigma}$ for deeper gaps. Once convolved, we expect the gap to become shallower and narrower, and we expect the effect of PSF smearing to depend on the ratio of

$$
\beta=\eta / w_{I},
$$

since with higher angular resolution (smaller $\eta$ ) and/or wider gaps (bigger $w_{I}$ ), the intrinsic gap profile at $\eta=0$ is better preserved, and vice versa. This is confirmed in the figure-for wider gaps, $\Delta_{I}(\eta)$ correlates well with $\Delta_{\Sigma}$ (as well as $\Delta_{I}(0)$ ), while narrower gaps become noticeably wider with finite $\eta$. For the depth, PSF smearing makes the gap shallower in all cases.

Quantitatively, we find that $\Delta_{I}(\eta)$ is correlated to $\Delta_{\Sigma}(\eta), \beta$, and $\delta_{I}$ in the following way:

$$
\Delta_{I}=\sqrt{\Delta_{\Sigma}^{2}+0.13 \frac{\beta^{2}}{\delta_{I}}}
$$



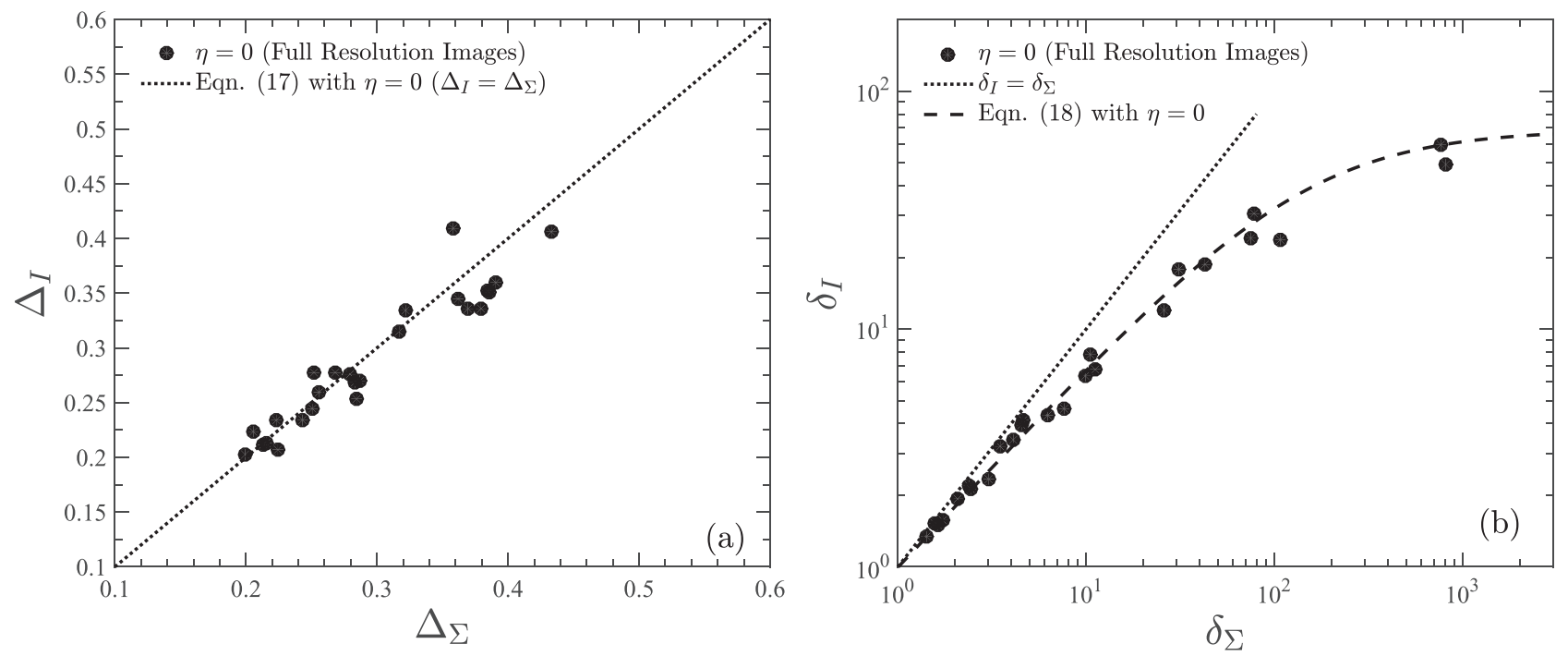

Figure 6. (a) Gap width in scattered light vs. gap width in surface density, and (b) gap depth in scattered light vs. gap depth in surface density. See Section 3.4 for details.
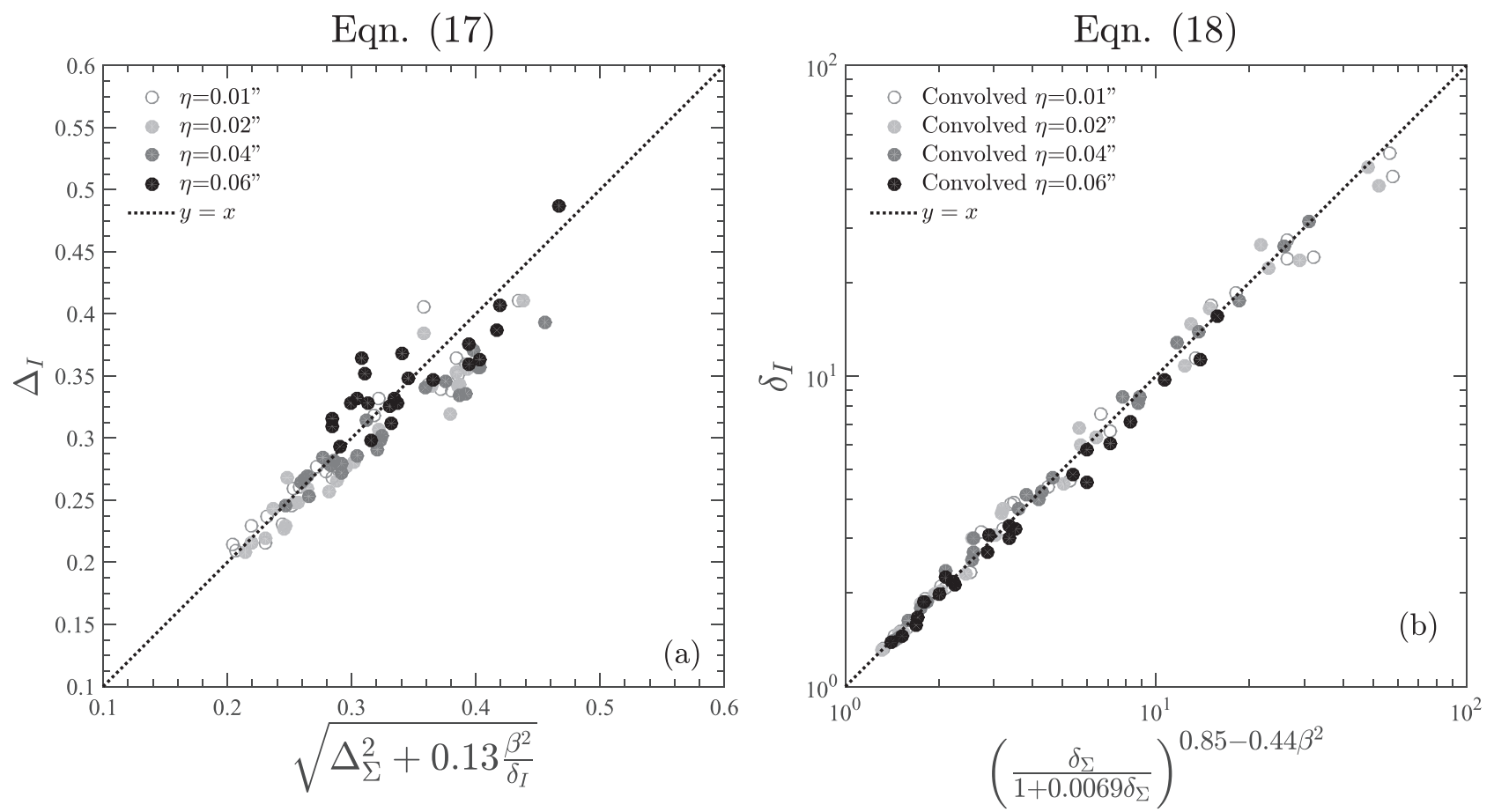

Figure 7. Effect of PSF smearing in gap width and depth, showing the correlations described by Equations (20) and (21). See Section 3.4 for details.

with $\sigma=9 \%$. In the high angular resolution limit $(\beta \rightarrow 0)$, $\Delta_{I} \rightarrow \Delta_{\Sigma}$. For the gap depth, we find

$$
\delta_{I}=\left(\frac{\delta_{\Sigma}}{1+0.0069 \delta_{\Sigma}}\right)^{0.85-0.44 \beta^{2}},
$$

with $\sigma=20 \%$. In the high angular resolution $(\beta \rightarrow 0)$ and shallow gap limit $\left(\delta_{\Sigma} \rightarrow 1\right), \delta_{I} \rightarrow \delta_{\Sigma}$. Both equations are invertible, and it is straightforward to obtain $\delta_{\Sigma}$ and $w_{\Sigma}$, given $\delta_{I}, w_{I}$, and $\eta$. These correlations are synthesized for the parameter space of $0.2 \lesssim \Delta_{I} \lesssim 0.5$ and $1 \lesssim \delta_{I} \lesssim 50$; we caution their applications to the parameter space beyond.

\subsection{Effects of Inclinations}

In this section, we compare the radial profile of face-on images to the radial profile along the major axis of disks at modest inclinations $\left(i \lesssim 45^{\circ}\right)$. In the latter, the radial profiles are averaged over a wedge of $\pm 10^{\circ}$ from the major axis centered on the central star, as indicated in Figure 8(c). We note that in inclined disks, equal distance points from the center fall approximately on ellipses not centered on the star (shifted along the minor axis), because these ellipses are at non-zero height (Figure 1 in Stolker et al. 2016b). Therefore, strictly speaking the major axis going through the star is not the major axis of the gap ellipse (the two are parallel to each other). Nevertheless, measuring the radial profile along the 

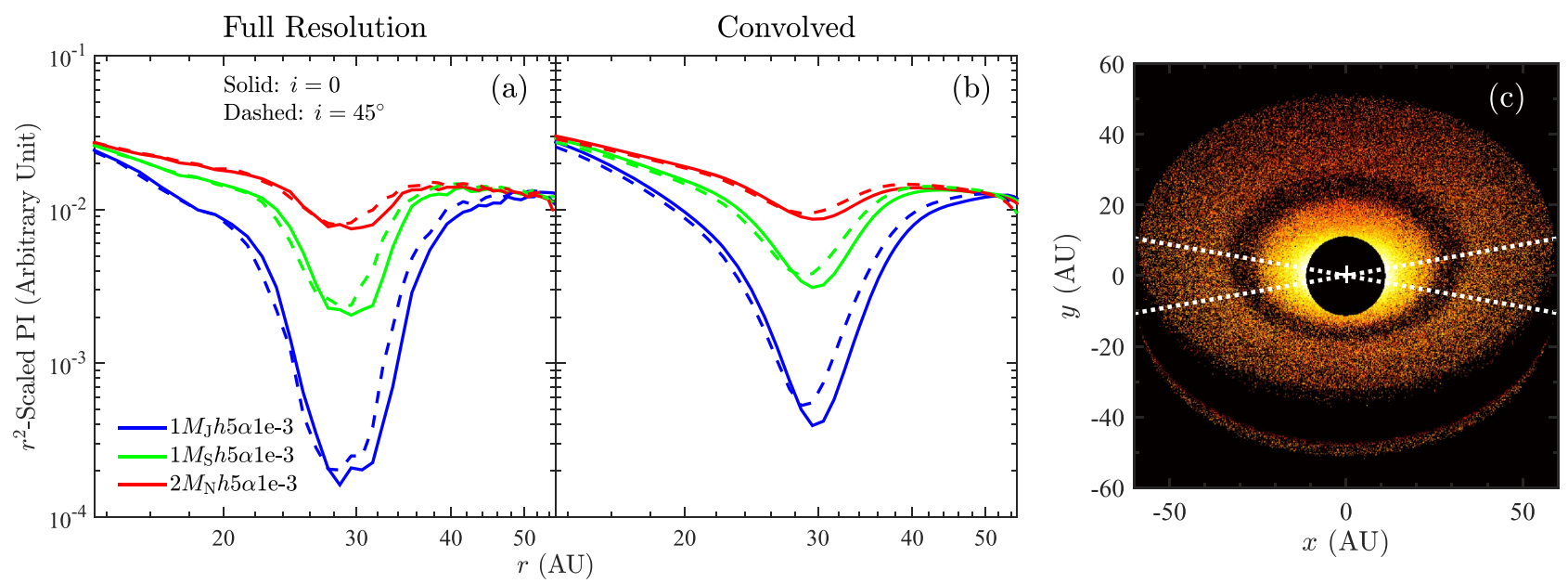

Figure 8. Radial profiles of three representative models with $h / r=0.05, \alpha=10^{-3}$, and $M_{\mathrm{p}}=1 M_{\mathrm{J}}, 1 M_{\mathrm{S}}$, and $2 M_{\mathrm{N}}$ (red, green, and blue curves, respectively). Solid curves are azimuthally averaged radial profiles for face-on images; dashed curves are the radial profiles along the major axis at $i=45^{\circ}$, averaged over a wedge with an opening angle of $20^{\circ}$, as indicated in panel (c). Panels (a) and (b) are for full-resolution and convolved images, respectively. All $i=45^{\circ}$ radial profiles have been scaled by the same constant factor to meet the solid curves at the same point at 12 and 55 au. Radial profiles at $45^{\circ}$ are very similar to radial profiles at face-on. See Section 3.5 for details.

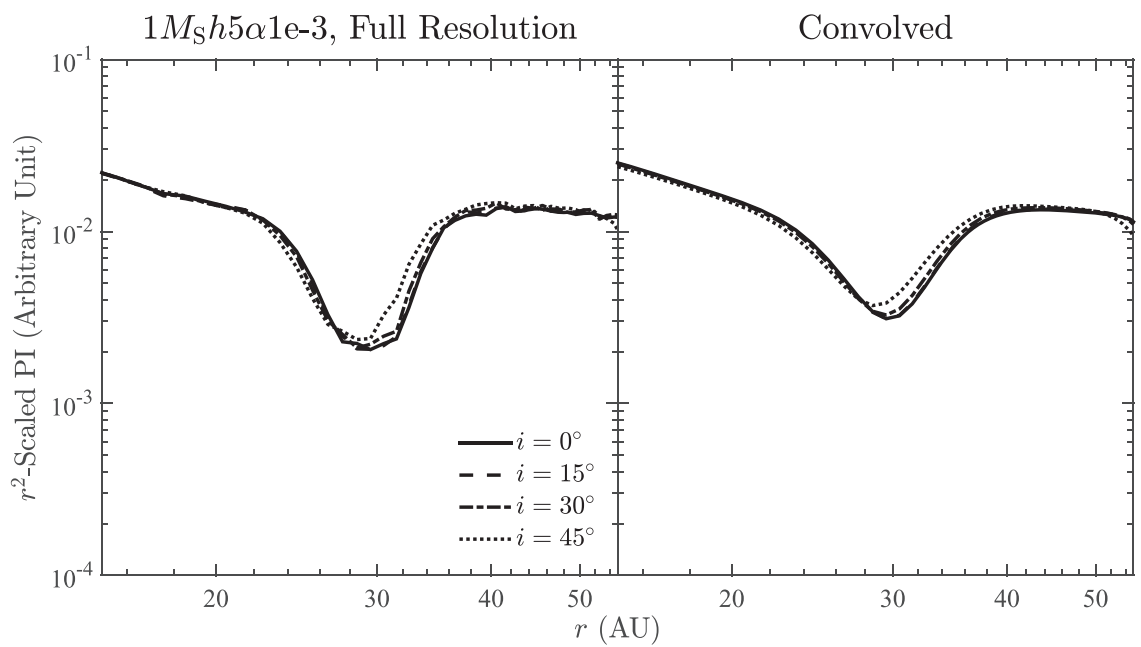

Figure 9. Radial profiles of the $1 M_{\mathrm{S}} h 5 \alpha 1 \mathrm{e}-3$ model in full resolution (left) and with $\eta=0 ! .04$ (right) at four different inclinations. For $i=0$ radial profiles are azimuthally averaged, while at finite $i$ radial profiles are averaged over a wedge with an opening angle of $20^{\circ}$ (Figure $8(\mathrm{c}$ )). The curves with $i \neq 0$ have been scaled so that they meet the $i=0^{\circ}$ profile at 12 and 55 au. The gap depth and width at $i \leqslant 45^{\circ}$ only weakly depend on inclinations. See Section 3.5 for details.

major axis centered on the star is common practice in the field (e.g., de Boer et al. 2016; Ginski et al. 2016); to facilitate the applications of our results, we do the same. The scattering angle along the major axis is close to $90^{\circ}$, the same as in face-on images. Therefore, the relative radial profile along the major axis is minimally affected by dust-scattering properties.

Major axis radial profiles at modest inclinations closely follow their face-on counterparts. Figure 8 compares the two for three representative models with $\alpha=10^{-3}, h / r=0.05$, and $M_{\mathrm{p}}=2 M_{\mathrm{N}}, 1 M_{\mathrm{S}}$, and $1 M_{\mathrm{J}}$. In all cases, the gap region in the inclined disk is slightly shifted inward compared with the faceon disk, due to the the inclination effect discussed above. The outer gap edge is shifted slightly more than the inner edge. Figure 9 shows the gap profile at $i=0^{\circ}, 15^{\circ}, 30^{\circ}$, and $45^{\circ}$ for both the full-resolution and convolved images of model $1 M_{\mathrm{S}} h 5 \alpha 1 \mathrm{e}-3$. Intuitively, the differences between the inclined and face-on images increase with $i$. When $i$ approaches $0^{\circ}$, the differences diminish.
Quantitatively, the gap width in inclined disks agree with face-on disks very well,

$$
\Delta_{I}\left(45^{\circ}\right)=\Delta_{I}(0),
$$

with a standard deviation of $5 \%$ between the two. On the other hand, inclined images have systematically shallower gaps,

$$
\delta_{I}(i)=\delta_{I}(0)\left(1-1.2 \times 10^{-6} i^{3}\right),
$$

with a standard deviation of $9 \%$ ( $i$ is in units of degrees). The effect of inclination on $\delta_{I}(i)$ is weak-with $i=45^{\circ}$, the gap depth is only reduced by $10 \%$. Figure 10 compares $\Delta_{I}\left(45^{\circ}\right)$ versus $\Delta_{I}(0)$, and $\delta_{I}\left(45^{\circ}\right)$ versus $\delta_{I}(0)$ for six representative models.

\subsection{Effects of the Flareness of the Disk}

So far, $h / r$ is taken to be a constant in our models (i.e., no radial dependence). This choice results in a flat disk surface, which simplifies the problem in our qualitative analysis of the 

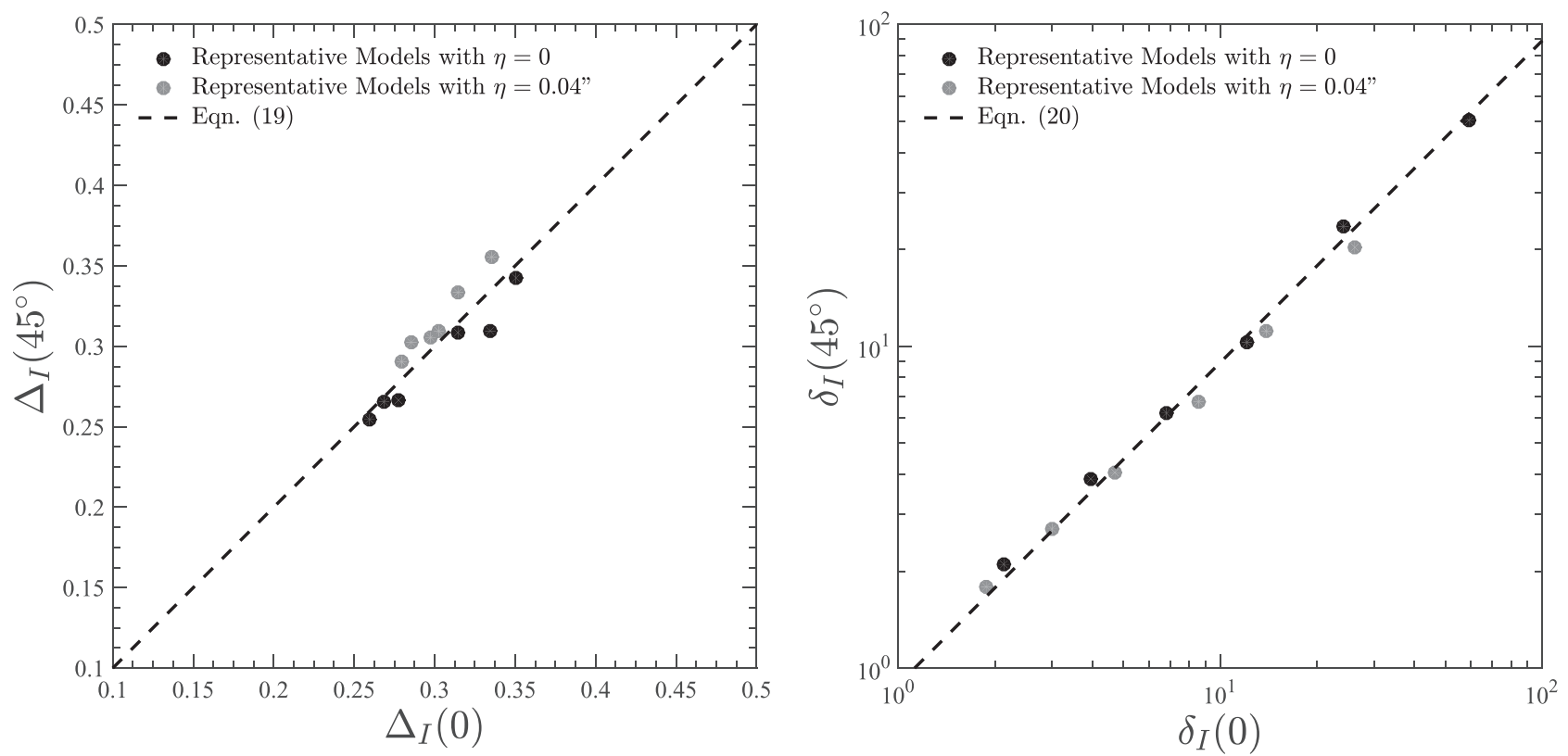

Figure 10. Gap width and depth in inclined disks. Panel (a) shows the gap width at $i=45^{\circ}$ vs. $i=0$ for six representative models for both full-resolution (black points) and convolved images (gray points). The models are $1 M_{\mathrm{J}} h 5 \alpha 3 \mathrm{e}-3,1 M_{\mathrm{J}} h 5 \alpha 1 \mathrm{e}-3,1 M_{\mathrm{J}} h 7 \alpha 1 \mathrm{e}-3,1 M_{\mathrm{S}} h 5 \alpha 1 \mathrm{e}-3,2 M_{\mathrm{N}} h 5 \alpha 1 \mathrm{e}-3$, and $2 M_{\mathrm{N}} h 5 \alpha 3 \mathrm{e}-4$ Panel (b) shows the gap depth at $i=45^{\circ}$ vs. $i=0$ for these models. Correlations (22) and (23) are overplotted.

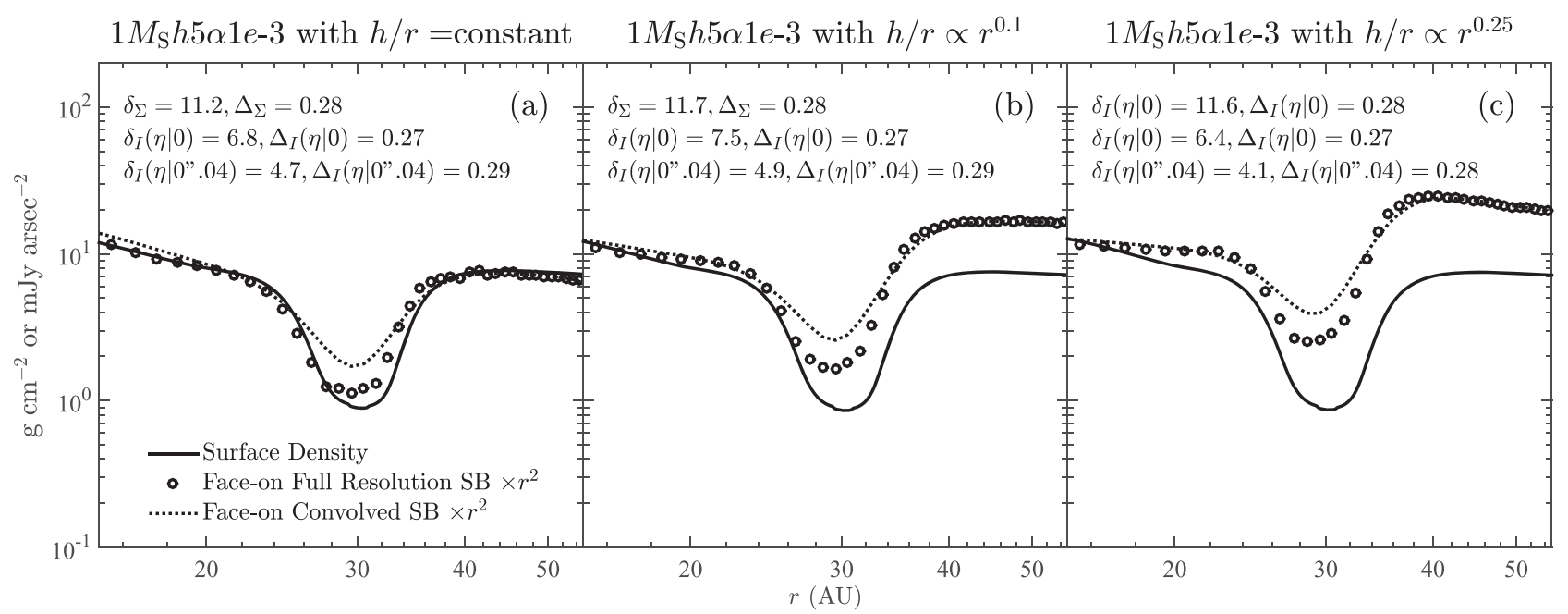

Figure 11. Radial profiles of the surface density (solid) and $H$-band PI surface brightness (circles: full resolution; dotted line: convolved; both scaled by $r^{2}$ ) of three models with $h / r=0.05$ at $r_{\mathrm{p}}=30 \mathrm{au}, \alpha=10^{-3}, M_{\mathrm{p}}=1 M_{\mathrm{S}}$, and different radius dependence of $h / r$ : (a) $h / r$ is a constant (flat disk; our fiducial setting), (b) $h / r \propto r^{0.1}$, (c) $h / r \propto r^{0.25}$. The disk in (b) and (c) is flared. Both the full-resolution and convolved surface brightness curves in each panel have been scaled by the same constant factor so that they roughly meet the surface density curves at $r=15 \mathrm{au}$. As the disk becomes more flared, the outer disk becomes brighter, as expected. However, the width and depth of the gap in both the surface density and scattered light (measurements printed in each panel) stay roughly the same, as the gap is approximately a local structure, and thus not significantly affected by the global flareness of the disk. See Section 3.6 for details.

physical picture, and enables us to obtain a straightforward intuition why scattered-light features closely follow the structures in surface density (Section 3.1). However, real disks passively heated by the central star may be flared, with $h / r \propto r^{\gamma}$ and $\gamma>0$ (e.g., Chiang \& Goldreich 1997). For example, by modeling the spectral energy distribution (SED) and resolved mm observations, Andrews et al. (2011) obtained $\gamma=0.19 \pm 0.1$ for a sample of 12 transitional disks. This surface flareness certainly affects the scattered-light distribution in disks; for example, flared disks have surface brightness decreasing slower with radius than flat disks. This may affect the width and depth of gaps in images, and slightly alter the surface density profiles (through Equations (16) and (17)).
However, the narrow gaps explored in this study are essentially local structures. In this case, the $h / r$ variation across the gap under a reasonable $\gamma$ (e.g., $\gamma \lesssim 0.25$ ) may be too small to produce any significant effect. To test this hypothesis, we carry out experiments and compare three models with different $\gamma$ 's, but with otherwise the same parameters, to examine the dependence of the gap width and depth on $\gamma$. The results are shown in Figure 11. With $h / r=0.05$ at $r_{\mathrm{p}}=30$ au, $\alpha=10^{-3}$, and $M_{\mathrm{p}}=1 M_{\mathrm{S}}$, the three models with $\gamma=0$ (flat disk), 0.1 , and 0.25 (flare disks) produce very similar gap properties in both the surface density and scattered light (the global scattered-light profiles in these models are of course different-flared disks have a brighter outer disk-as 
expected). Specifically, for $\Delta_{\Sigma}$ and $\Delta_{I}$, all three models return essentially the same values; for $\delta_{\Sigma}$, the two flared models have measurements higher than the flat model by $\sim 5 \%$; and for $\delta_{I}(\eta \mid 0)$, the two flared models differ from the flat model by about $10 \%$. Therefore, we tentatively conclude that under reasonable flareness $(\gamma \lesssim 0.25)$, the flaring of the disk does not significantly affect the gap properties in both the density and image space for narrow gaps as in this example; subsequently, we expect the correlations derived in this paper to hold to a good approximation. A full parameter space exploration is needed to firmly establish this statement for more general cases.

\subsection{A Generic Roadmap to Link $\left[\Delta_{I}, \delta_{I}\right]$ with $\left[M_{\mathrm{p}}, h / r, \alpha\right]$}

Fitted correlations (16)-(23) compose a complete set of equations to link $\left[\Delta_{I}, \delta_{I}\right]$ with $\left[M_{\mathrm{p}}, h / r, \alpha\right]$. Once a narrow gap $\left(\Delta_{I}(\eta, i) \lesssim 0.5\right)$ is identified in the scattered light of a disk at a modest inclination $\left(i \lesssim 45^{\circ}\right)$ with both the inner and outer gap edges clearly revealed, one can follow the following steps to link the observed gap properties to the planet and disk parameters $\left[M_{\mathrm{p}}, h / r, \alpha\right]$ :

I. Use Equations (22)-(23) to eliminate the effect of inclination: $\delta_{I}(\eta, i) \rightarrow \delta_{I}(\eta, 0), \Delta_{I}(\eta, i) \rightarrow \Delta_{I}(\eta, 0)$;

II. Use Equations (19)-(21) to eliminate the effect of finite angular resolution, and link images to surface density: $\delta_{I}(\eta, 0) \rightarrow \delta_{\Sigma}, \Delta_{I}(\eta, 0) \rightarrow \Delta_{\Sigma}$

III. Finally, Equations (16) and (17) link the gap properties in surface density to $\left[M_{\mathrm{p}}, h / r, \alpha\right]$. Note that $h / r$ can normally be constrained directly from the gap width, and it leaves the quantity $q^{2} / \alpha$ to be constrained from the gap depth.

We note that these correlations are derived for PI at $H$-band, but they also apply to total intensity images and/or images in other spectral bands within a factor of $\sim 2$ difference in wavelength from $H$-band (as long as the signals are dustscattering dominated), because both depth and width are measured in a relative sense, and face-on radial profiles and major axis radial profiles in inclined images minimize the dependence on scattering angles. Finally, we emphasize that a key precondition when applying these correlations to actual systems is that the gap bottom needs to be detected (i.e., the gap bottom in images should be above the detection threshold). If not, the gap depth becomes ill-defined.

\subsection{Cautions when Applying Our Results and Possible Future Improvements}

In simulating the gap images and deriving the correlations, we have made a number of assumptions about the disk structures and in modeling. Here we comment on the effects of these assumptions, and caution the readers about the caveats when applying our results to real disks.

1. Jang-Condell \& Turner (2012, Figure 2) highlighted that the outer gap edge may receive extra stellar radiation due to the depletion of material inside the gap, leading to higher temperature, thus higher $h / r$ (see also JangCondell 2008; Isella \& Turner 2016). However, whether this effect can increase the contrast of the gap at the outer edge needs additional investigations. Fung \& Chiang (2016, Figure 6) showed that gas inside the gap and around the gap edges circulate, therefore the heating at the outer edge is redistributed to a much larger region in the disk, weakening this thermal feedback. Future coupled hydro-radiation-transfer simulations are needed to quantify this effect.

2. Ribas et al. (2014; see also Hernández et al. 2008) showed that the fraction of stars with protoplanetary disks, indicated by their infrared excesses, drops on a timescale of about $3 \mathrm{Myr}$. The timescale that a planet takes to fully open a gap with a width of $6 h$ (i.e., our gap width) is approximately the viscous timescale to cross $6 h$, $\tau_{\nu}=6 /\left(\alpha(h / r) \Omega_{\mathrm{K}}\right)$. With $\alpha(h / r) /\left(r_{\mathrm{p}} / 30 \mathrm{au}\right)^{1.5} \lesssim 10^{-4}$, $\tau_{\nu}$ is longer than $3 \mathrm{Myr}$. Therefore, in low viscosity disks, very young systems, and large separations from the center, gaps may not reach their final depth and width, which invalidates the $\left[M_{\mathrm{p}}, h / r, \alpha\right]-\left[\Delta_{\Sigma}, \delta_{\Sigma}\right]$ correlations synthesized in this work and in others. This gap-opening timescale issue is however irrelevant to the $\left[\delta_{I}, \Delta_{I}\right]-\left[\delta_{\Sigma}\right.$, $w_{\Sigma}$ ] conversions, and we expect them to hold even when the gap is only partially opened.

3. In our MCRT simulations, no noise is added into the images (apart from the intrinsic Poisson noise due to the finite number of photons), thus the gap bottom reaches its theoretical minimum. If in real observations the gap bottom reaches the noise level, the measured gap depth and the derived planet mass will be its lower limits, while the measured gap width and the derived disk scale height, will be its upper limits.

\section{Applications to the Gaps in HD 169142, TW HYA, HD 97048, LKCA 15, and RX J1615.3-3255}

As examples, in this section we apply our results to a few gaps imaged in actual systems (Figure 12; "gaps" in these systems refer to the regions around local minima on the $r^{2}$ scaled scattered-light profile). The gap bottom in these disks is robustly detected. Assuming each gap is opened by a single planet, we derive $h / r$ and $q^{2} / \alpha$ at the gap location, as well as the planet mass for several assumed $\alpha$. The results are summarized in Table 2. We stress that the derived disk and planet properties in the table should be taken as suggested values only, as real disks may not obey the assumptions in our models outlined in Section 3.8, and some of the gaps observed so far may or may not be opened by (a single) planet. Actual hydro+MCRT modeling of individual disks with planet-disk interaction models quantitatively fitting the observations is encouraged to more accurately recover the disk and planet properties in specific systems.

\section{1. $H D 97048$}

HD 97048 is a $\sim 2.5 M_{\odot}$ Herbig Ae/Be star located at $\sim 158$ pc (van den Ancker et al. 1998; van Leeuwen 2007) surrounded by a protoplanetary disk several hundreds of astronomical units in size. The disk is inclined at $\sim 40^{\circ}$ based on mm observations (van der Plas et al. 2017; Walsh et al. 2016). Maaskant et al. (2013) first suggested that this disk may be gap-/cavity-harboring based on SED modeling. Very recently, VLT/SPHERE $J$-band imaging in both polarized and total intensity found multiple rings and gaps in this system (Ginski et al. 2016). In addition, the $\mathrm{mm}$ dust continuum counterparts of some of these structures may have been found by van der Plas et al. (2017), suggesting the scattered-light gaps and rings are likely to be physical density structures instead of shadow effects. 
HD 97048 (SPHERE $J$-band) TW Hya (SPHERE $H$-band) HD 169142 (HiCIAO $H$-band) LkCa 15 (SPHERE $J$-band) Ginski et al. 2016

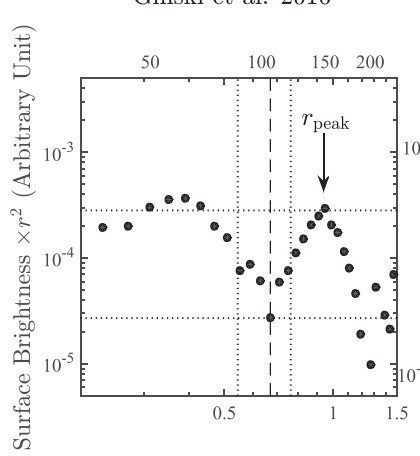

van Boekel et al. 2016

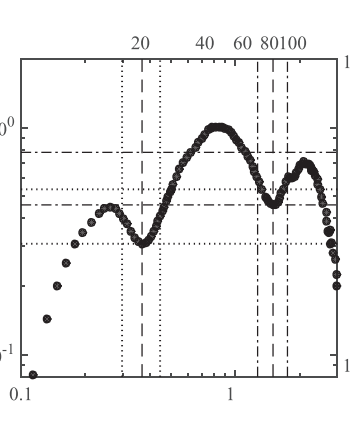

Momose et al. 2015 $r(\mathrm{AU})$
J1615 (SPHERE $J$-band) de Boer et al. 2016

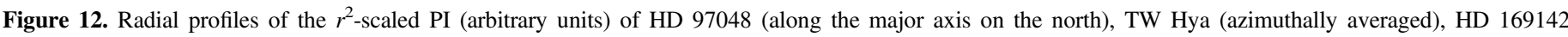

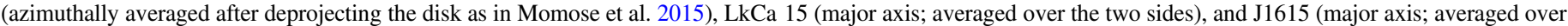

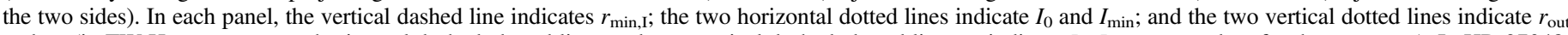

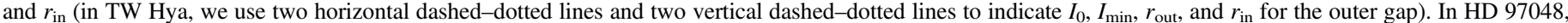

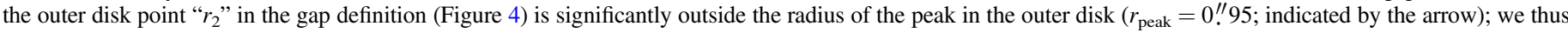
fix $r_{2}=r_{\text {peak }}$ as the "outside the gap" point. $r_{\text {out }}, r_{\text {in }}, I_{0}$, and $I_{\min }$ are listed in Table 2. See Section 4 for details.

Table 2

Applying Our Results to Six Gaps Detected in Scattered Light

\begin{tabular}{|c|c|c|c|c|c|c|}
\hline & $\begin{array}{c}\text { HD } 97048^{\mathrm{a}} \\
\text { Ginski et al. (2016) }\end{array}$ & $\begin{array}{c}\text { TW Hya }{ }^{\mathrm{b}} \\
\text { van Boekel } \\
\text { et al. }(2016)\end{array}$ & $\begin{array}{c}\text { TW Hya }{ }^{c} \\
\text { van Boekel } \\
\text { et al. }(2016)\end{array}$ & $\begin{array}{l}\text { HD } 169142^{\mathrm{d}} \\
\text { Momose } \\
\text { et al. }(2015)\end{array}$ & $\begin{array}{l}\text { LkCa15 } \\
\text { Thalmann } \\
\text { et al. (2016) }\end{array}$ & $\begin{array}{l}\text { RX J1615 } \\
\text { de Boer } \\
\text { et al. (2016) }\end{array}$ \\
\hline Band & $J$ & $H$ & $H$ & $H$ & $J$ & $J$ \\
\hline Radial Profile $^{\mathrm{f}}$ & Major Axis & Azim-Averaged & Azim-Averaged & Azim-Averaged & Major Axis & Major Axis \\
\hline$d$ & $158 \mathrm{pc}$ & $54 \mathrm{pc}$ & $54 \mathrm{pc}$ & $145 \mathrm{pc}$ & $140 \mathrm{pc}$ & $185 \mathrm{pc}$ \\
\hline$i$ & $40^{\circ}$ & $7^{\circ}$ & $7^{\circ}$ & $13^{\circ}$ & $50^{\circ}$ & $45^{\circ}$ \\
\hline$M_{\star}\left(M_{\odot}\right)$ & 2.5 & 0.8 & 0.8 & 2 & 1 & 1.1 \\
\hline$r_{\min , \mathrm{I}}$ & 0.' 67 (106 au) & $0.37(20 \mathrm{au})$ & $1 . .5(81 \mathrm{au})$ & $0.37(54 \mathrm{au})$ & $0.26(36 \mathrm{au})$ & $0.52(97 \mathrm{au})$ \\
\hline$w_{I}$ & $0 . " 22$ & 0.15 & 0.48 & $0 ! 17$ & 0.10 & 0.17 \\
\hline$\Delta_{I}$ & 0.33 & 0.41 & 0.32 & 0.47 & 0.41 & 0.34 \\
\hline$\delta_{I}$ & 10.4 & 1.7 & 1.7 & 2.1 & 3.6 & 2.5 \\
\hline$\eta^{\mathrm{g}}$ & $0 . " 04$ & 0.05 & $0 . " 05$ & 0.06 & 0.05 & 0.04 \\
\hline$\beta$ & 0.18 & 0.33 & 0.10 & 0.35 & 0.48 & 0.23 \\
\hline$\Delta_{I}(i \mid 0)$ & 0.33 & 0.41 & 0.32 & 0.47 & 0.41 & 0.34 \\
\hline$\delta_{I}(i \mid 0)$ & 11.3 & 1.7 & 1.7 & 2.1 & 4.2 & 2.8 \\
\hline$\Delta_{\Sigma}$ & 0.33 & 0.40 & 0.32 & 0.46 & 0.40 & 0.34 \\
\hline$\delta_{\Sigma}$ & 21 & 2.0 & 1.9 & 2.5 & 7.2 & 3.5 \\
\hline$h / r^{\mathrm{h}}$ & 0.056 & 0.068 & 0.055 & 0.079 & 0.069 & 0.058 \\
\hline$q^{2} / \alpha$ & $2.6 \times 10^{-4}$ & $3.6 \times 10^{-5}$ & $1.1 \times 10^{-5}$ & $1.1 \times 10^{-4}$ & $2.2 \times 10^{-4}$ & $3.8 \times 10^{-5}$ \\
\hline$M_{\mathrm{p}} / M_{\mathrm{J}}\left(\alpha=10^{-2}\right)^{\mathrm{i}}$ & 4.0 & 0.48 & 0.26 & 2.1 & 1.5 & 0.68 \\
\hline$M_{\mathrm{p}} / M_{\mathrm{J}}\left(\alpha=10^{-3}\right)$ & 1.3 & 0.15 & 0.08 & 0.67 & 0.47 & 0.22 \\
\hline$M_{\mathrm{p}} / M_{\mathrm{J}}\left(\alpha=10^{-4}\right)$ & 0.40 & 0.05 & 0.03 & 0.21 & 0.15 & 0.07 \\
\hline
\end{tabular}

Notes. The radial profiles of the gaps are shown in Figure 12.

a The "gap 2" in Ginski et al. (2016, Figure 2).

b The 21 au gap in van Boekel et al. (2016).

c The $\sim 85$ au gap in van Boekel et al. (2016).

d The 40-70 au gap in Momose et al. (2015).

e The gap structure "G" in Boer et al. (2016, Figure 1).

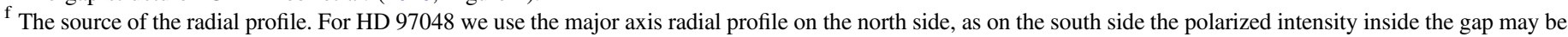

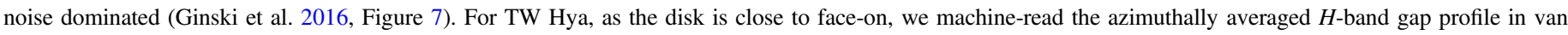

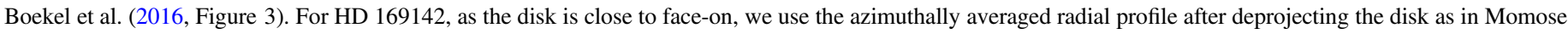

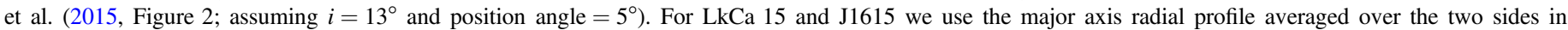
Thalmann et al. (2016, Figure 4(a); Deep) and de Boer et al. (2016, Figure 7).

Angular resolution of the observation as the FWHM of the PSF in the actual data.

h Derived $h / r$ at the location of the gap.

${ }^{\mathrm{i}}$ Planet mass assuming the disk viscosity in the parentheses. See Section 4 for details.

Here we focus on the "gap 2" in the $J$-band VLT/SPHERE data set (Ginski et al. 2016). Comparing with the other gaps, gap 2 is well detected in both polarized and total intensity, with the inner and outer edges clearly resolved. We obtain $h / r=0.06$ at the location of the gap $(0$ " $67 ; 106 \mathrm{au})$, and constrain the planet mass to be between $0.4-4 M_{\mathrm{J}}$ for 
$10^{-4} \lesssim \alpha \lesssim 10^{-2}$. By analyzing the offset of the center of the gap ellipse from the star, Ginski et al. (2016) determined that at the gap location the disk's surface is at $\theta \sim 0.2 \mathrm{rad}$. Therefore, the disk surface is about 3-4 scale heights away from the disk midplane, which is reasonable (e.g., Chiang \& Goldreich 1997; we note that in our MCRT simulations the disk surface is also generally about 4 scale heights away from the midplane). Ginski et al. (2016) ruled out the presence of planets more massive than $2 M_{\mathrm{J}}$ at the gap location (assuming the BT-SETTL isochrones; Allard et al. 2011). Comparing with our constraints on the planet mass, this suggests that the viscosity in the gap may be low, $\alpha \lesssim 10^{-3}$.

\section{2. $T W H y a$}

TW Hya is a $\sim 0.8 M_{\odot}$ K6 star located at 54 pc (Torres et al. 2006, 2008). The nearly face-on disk $\left(i \sim 7^{\circ}\right.$; Qi et al. 2004) has been imaged by HST (Debes et al. 2013, 2016), Gemini/GPI (Rapson et al. 2015b), Subaru/HiCIAO (Akiyama et al. 2015), and VLT/SPHERE (van Boekel et al. 2016), and multiple gaps have been identified in scattered light: one at $\sim 80 \mathrm{au}$, one at $\sim 20 \mathrm{au}$, and one at $\lesssim 6 \mathrm{au}$. The $\mathrm{mm}$ continuum counterpart of the 20 au gap may have been identified by Andrews et al. (2016) in ALMA observations at a slightly larger radius (possibly caused by the dust filtration effect).

Here we examine two gaps in the system: the one at $\sim 20$ au and the one at $\sim 80 \mathrm{au}$, in the $H$-band VLT/SPHERE data set (van Boekel et al. 2016, Figure 3). Due to the low inclination, the azimuthally averaged radial profile is used. For the inner gap, $h / r$ at the gap location $(0 . .37 ; 20 \mathrm{au})$ is 0.068 , and the planet mass is between 0.05 and $0.5 M_{\mathrm{J}}$ for $10^{-4} \lesssim \alpha \lesssim 10^{-2}$; for the outer gap, $h / r$ at the gap location $\left(1{ }^{\prime \prime} 5 ; 81 \mathrm{au}\right)$ is 0.055 , and the planet mass is between $0.03-0.3 M_{\mathrm{J}}$ for $10^{-4} \lesssim \alpha \lesssim 10^{-2}$.

Using hydro+MCRT simulations, Rapson et al. (2015b) tentatively fit the observed gap profile at $\sim 20$ au in the Gemini/ GPI data set using a $0.16 M_{\mathrm{J}}$ planet at $21 \mathrm{au}$, assuming $\alpha=10^{-3}$ and $h / r=0.068$. Their results agree well with our findings. van Boekel et al. (2016) used radiative transfer simulations to transform the scattered-light map into surface density and scale height profiles. At $20 \mathrm{au}$ they obtained $h / r=0.05$, while at $80 \mathrm{au}$ they obtained $h / r=0.08$. Subsequently, assuming $\alpha=2 \times 10^{-4}$, they derived the masses of the planets at 20 au and $80 \mathrm{au}$ to be $0.05 M_{\mathrm{J}}$ and $0.11 M_{\mathrm{J}}$, based on a similar version of Equation 17 in Duffell (2015). While their $h / r$ and $M_{\mathrm{p}}$ at the inner gap are broadly consistent with our results, our estimated $h / r$ for the outer gap is significantly lower (and also lower than our estimated $h / r$ at $20 \mathrm{au}$ ), resulting in a lower estimate for $M_{\mathrm{p}}$ as well. This may indicate that the 80 au gap is not opened by a planet; alternatively, this may be because our assumption of the gap structure being local is no longer valid for the 80 au gap, which may lie in the shadow created by the inner disk and thus the underlying "background" is no longer smooth.

\section{3. $H D 169142$}

HD 169142 is a $\sim 2 M_{\odot}$ Herbig Ae star located at $145 \mathrm{pc}$ (Sylvester et al. 1996; Raman et al. 2006). It has a prominent protoplanetary disk at an inclination of $13^{\circ}$, determined from gas kinematics (Raman et al. 2006; Panić et al. 2008). A narrow gap around 50 au was first discovered in $H$-band VLT/
NACO polarized light imaging (Quanz et al. 2013), and subsequently found by Subaru/HiCIAO (Momose et al. 2015) and in dust thermal emission at $7 \mathrm{~mm}$ by VLA (Osorio et al. 2014). The radius-varying disk profile was interpreted as two power laws in the inner and outer disks joined by a transition region in between by Momose et al. (2015). Osorio et al. (2014) also discovered a blob in the $7 \mathrm{~mm}$ VLA data set residing right inside the gap, with an estimated total mass of $6 \times 10^{-6} M_{\odot}$ in dust. Through radiative transfer modeling, Wagner et al. (2015) determined that this gap cannot be explained by shadow effects caused by the inner disk, leaving the planet-sculpting scenario as a favorite hypothesis. By comparing the apparent gap depth in $7 \mathrm{~mm}$ observations with their $M_{\mathrm{p}}-\delta_{\Sigma}$ relation, Kanagawa et al. (2015) estimated the mass of the putative planet to be $M_{\mathrm{p}} \gtrsim 0.4 M_{\mathrm{J}}$. As Rosotti et al. (2016) pointed out, such estimate is risky as mm observations trace mm-sized grains, which can have a substantially different spatial distribution from the gas due to dust/gas coupling effects. The disk has another inner gap at $\sim 25$ au (Honda et al. 2012; Quanz et al. 2013), and a companion candidate at the edge of the inner gap (Reggiani et al. 2014; see also Biller et al. 2014).

Here we examine the gap at 40-70 au in the Subaru/ HiCIAO $H$-band data set (Momose et al. 2015). Due to the low inclination, we adopt an azimuthally averaged radial profile after deprojecting the disk (Momose et al. 2015, Figure 2; assuming $i=13^{\circ}$ and position angle $=5^{\circ}$ ). We derive $h / r$ at the gap location $(0 . / 35 ; 51 \mathrm{au})$ to be 0.08 , and the planet mass to be between $0.2-2.1 M_{\mathrm{J}}$ for $10^{-4} \lesssim \alpha \lesssim 10^{-2}$.

\section{4. $\operatorname{LkCa} 15$}

LkCa 15 is a $\sim 1 M_{\odot} \mathrm{K} 3$ star located at $140 \mathrm{pc}$ (Simon et al. 2000; Piétu et al. 2007). Using Spitzer IRS spectrum modeling, Espaillat et al. (2010) identified the system as a transitional disk with an outer disk, an inner disk, and a gap in between. SMA $\mathrm{mm}$ observations resolved the gap and determined its outer radius to be $50 \mathrm{au}$ in dust continuum emission (Andrews et al. 2011). The gap has since been resolved in scattered light by Subaru/HiCIAO (Thalmann et al. 2010), Gemini/NICI (Thalmann et al. 2014), and VLT/SPHERE (Thalmann et al. $2015,2016)$ in both total intensity and PI. The latest VLT/ SPHERE observations by Thalmann et al. (2016) at multiple optical to NIR bands clearly showed that the system has a substantial inner disk, and the gap is narrow enough $\left(\Delta_{I}<0.5\right)$ to warrant the hypothesis that it may be opened by a single planet. The inclination of the disk is $\sim 50^{\circ}$ based on $\mathrm{mm}$ observations (Piétu et al. 2007; Andrews et al. 2011; van der Marel et al. 2015).

Here we examine the gap profile along the major axis of the disk in the VLT/SPHERE $H$-band PI data set presented in Thalmann et al. (2016). We derive $h / r$ at the gap location $(0$ ". $26 ; 36 \mathrm{au})$ to be 0.07 , and the planet mass to be between $0.15-1.5 M_{\mathrm{J}}$ for $10^{-4} \lesssim \alpha \lesssim 10^{-2}$.

LkCa has several detected planet candidates. Using nonredundant aperture masking interferometry on Keck, Kraus \& Ireland (2012) identified a point source at a deprojected distance of $\sim 20$ au from the star. Recently, Sallum et al. (2015) reported the detections of two additional point sources in the system with LBT/LBTI and Magellan/MagAO. The three planet candidates in Sallum et al. (2015) are located between 15 and $19 \mathrm{au}$. We note that these planet candidates are unlikely to be the one responsible for maintaining the gap edge in scattered 
light at $\sim 50$ au (i.e., the one whose properties we are inferring here), as they are too far away from the gap edge. The radial profile of the gap (Figure 12(d)) is not inconsistent with the gap being opened by just one planet. Additional planets at $r \lesssim 20$ au with sufficiently low mass will not significantly affect the gap opened by the outer planet.

\section{5. $R X J 1615.3-3255$}

RX J1615.3-3255 (hereafter J1615) is a $1.1 M_{\odot}$ K5 star at 185 pc (Wichmann et al. 1997; Wahhaj et al. 2010; Andrews et al. 2011). The system has a gap with an outer radius of $\sim 20-30$ au, revealed in $\mathrm{mm}$ dust continuum emission first by SMA (Andrews et al. 2011) and subsequently by ALMA (van der Marel et al. 2015). The disk has a modest inclination, $i \sim 45^{\circ}$. Recently, de Boer et al. (2016) resolved this system in scattered light at multiple optical-to-NIR wavelengths using VLT/SPHERE, and identified multiple gaps and rings in the system.

Here we examine the the major axis profile of the gap at $\sim 90$ au in the $J$-band VLT/SPHERE data set presented by de Boer et al. (2016, marked "G" in Figure 1). We derive $h / r$ at the gap location $\left(0\right.$ "' between $0.07-0.7 M_{\mathrm{J}}$ for $10^{-4} \lesssim \alpha$ $\lesssim 10^{-2}$.

By analyzing the alternating bright/dark pattern on the rings, de Boer et al. (2016) found tentative evidence to suggest that the rings in $\mathrm{J} 1615$ might be caused by shadows (i.e., variation in scale height instead of surface density). At the moment the evidence is inconclusive.

\section{Summary}

Combining 2D and 3D hydro simulations with 3D radiative transfer simulations, we examine the morphology of planetopened gaps in near-infrared scattered-light images. Quantitatively, we obtain correlations between gap depth and width in inclined disks with finite angular resolution and the intrinsic gap depth and width in face-on images with infinite resolution. The latter is subsequently quantitatively linked to the gap depth and width in disk surface density assuming a parameterized $h / r$ profile across the gap region, which can be used to constraints the mass of the gap-opening planet mass $M_{\mathrm{p}}$, the disk scale height at the location of the gap $h / r$, and disk viscosity $\alpha$. The main takeaways are

1. 2D hydro simulations, puffed up using an assumed midplane temperature profile, produce the same gap profile in scattered-light images as 3D simulations.

2. With our definition illustrated in Figure 4, the aspect ratio $h / r$ in the gap region can be directly backed out from the gap width. This can be used to constrain the midplane temperature in disks.

3. Equations (16)-(23) compose a complete set of correlations to link the observed $\left[\Delta_{I}, \delta_{I}\right]$ to $\left[M_{\mathrm{p}}, h / r, \alpha\right]$ for narrow gaps $\left(\Delta_{I} \lesssim 0.5\right)$ in disks with modest inclinations $\left(i \lesssim 45^{\circ}\right)$ and flareness $\left(h / r \propto r^{<0.25}\right)$. Once such a gap is identified in scattered light with both the inner and outer gap edges clearly revealed, one can follow the steps outlined in Section 3.7 to constrain the fundamental planet and disk parameters $M_{\mathrm{p}}, h / r$, and $\alpha$.

4. We apply our results to the gaps imaged in scattered light in HD 97048, TW Hya, HD 169142, LkCa 15, and RX J1615.3-3255 to derive $h / r$ and $M_{\mathrm{p}}^{2} / \alpha$ at the locations of their gaps. The results are listed in Table 2 (see also
Figure 12). Assuming $\alpha=10^{-3}$, the masses of all gapopening planets are roughly between 0.1 and $1 M_{\mathrm{J}}$.

We thank Eugene Chiang, Roman Rafikov, Sascha Quanz, Avenhaus Henning, and Tomas Stolker for motivating this work; Munetake Momose for sharing with us the SEEDS images of HD 169142; Sascha Quanz for sharing with us the VLT/NACO image of HD 169142; Joel Kastner and Valerie Rapson for sharing with us the Gemini/GPI image of TW Hya; Christian Ginski and Tomas Stolker for sharing with us the VLT/SPHERE data of HD 97048; Christian Thalmann for sharing with us the VLT/SPHERE LkCa 15 data on HD 97048; Jos de Boer for sharing with us the VLT/SPHERE data on RX J1615.3-3255; and Andrew Youdin for insightful discussions. We also thank the referee, Takayuki Muto, for constructive suggestions that greatly improved the quality of the paper. This project is supported by NASA through Hubble Fellowship grant HST-HF-51320.01-A (R.D.) awarded by the Space Telescope Science Institute, which is operated by the Association of Universities for Research in Astronomy, Inc., for NASA, under contract NAS 5-26555. J.F. gratefully acknowledges support from the Natural Sciences and Engineering Research Council of Canada, the Center for Integrative Planetary Science at the University of California, Berkeley, and the Sagan Fellowship Program funded by NASA under contract with the Jet Propulsion Laboratory (JPL) and executed by the NASA Exoplanet Science Institution.

\section{References}

Akiyama, E., Muto, T., Kusakabe, N., et al. 2015, ApJL, 802, L17

Allard, F., Homeier, D., \& Freytag, B. 2011, in ASP Conf. Ser. 448, 16th Cambridge Workshop on Cool Stars, Stellar Systems, and the Sun, ed. C. Johns-Krull, M. K. Browning, \& A. A. West (San Francisco, CA: ASP), 91

ALMA Partnership, Brogan, C. L., Pérez, L. M., et al. 2015, ApJL, 808, L3 Andrews, S. M., Wilner, D. J., Espaillat, C., et al. 2011, ApJ, 732, 42

Andrews, S. M., Wilner, D. J., Zhu, Z., et al. 2016, ApJL, 820, L40

Baraffe, I., Chabrier, G., Allard, F., \& Hauschildt, P. H. 1998, A\&A, 337, 403

Beuzit, J.-L., Feldt, M., Dohlen, K., et al. 2008, Proc. SPIE, 7014, 701418

Biller, B. A., Males, J., Rodigas, T., et al. 2014, ApJL, 792, L22

Bowler, B. P. 2016, PASP, 128, 102001

Brandt, T. D., McElwain, M. W., Turner, E. L., et al. 2014, ApJ, 794, 159

Carrasco-González, C., Henning, T., Chandler, C. J., et al. 2016, ApJL, 821, L16

Chiang, E. I., \& Goldreich, P. 1997, ApJ, 490, 368

Cumming, A., Butler, R. P., Marcy, G. W., et al. 2008, PASP, 120, 531

D’Angelo, G., Lubow, S. H., \& Bate, M. R. 2006, ApJ, 652, 1698

Debes, J. H., Jang-Condell, H., \& Schneider, G. 2016, ApJL, 819, L1

Debes, J. H., Jang-Condell, H., Weinberger, A. J., Roberge, A., \& Schneider, G. 2013, ApJ, 771, 45

de Boer, J., Salter, G., Benisty, M., et al. 2016, A\&A, 595, A114

Dodson-Robinson, S. E., \& Salyk, C. 2011, ApJ, 738, 131

Dong, R., \& Dawson, R. 2016, ApJ, 825, 77

Dong, R., Fung, J., \& Chiang, E. 2016, ApJ, 826, 75

Dong, R., \& Fung, J. 2016, ApJ, in press (arXiv:1612.00446)

Dong, R., Rafikov, R., Zhu, Z., et al. 2012, ApJ, 750, 161

Dong, R., Rafikov, R. R., \& Stone, J. M. 2011a, ApJ, 741, 57

Dong, R., Rafikov, R. R., Stone, J. M., \& Petrovich, C. 2011b, ApJ, 741, 56

Dong, R., Zhu, Z., \& Whitney, B. 2015, ApJ, 809, 93

Duffell, P. C. 2015, ApJL, 807, L11

Duffell, P. C., \& Dong, R. 2015, ApJ, 802, 42

Duffell, P. C., \& MacFadyen, A. I. 2013, ApJ, 769, 41

Espaillat, C., D’Alessio, P., Hernández, J., et al. 2010, ApJ, 717, 441

Follette, K. B., Grady, C. A., Swearingen, J. R., et al. 2015, ApJ, 798, 132

Fouchet, L., Gonzalez, J.-F., \& Maddison, S. T. 2010, A\&A, 518, A16

Fung, J. 2015, PhD thesis, Univ. Toronto

Fung, J., \& Chiang, E. 2016, ApJ, 832, 105

Fung, J., Shi, J.-M., \& Chiang, E. 2014, ApJ, 782, 88

Ginski, C., Stolker, T., Pinilla, P., et al. 2016, A\&A, 595, A112 
Gonzalez, J.-F., Pinte, C., Maddison, S. T., Ménard, F., \& Fouchet, L. 2012, A\&A, 547, A58

Goodman, J., \& Rafikov, R. R. 2001, ApJ, 552, 793

Hashimoto, J., Dong, R., Kudo, T., et al. 2012, ApJL, 758, L19

Hashimoto, J., Tamura, M., Muto, T., et al. 2011, ApJL, 729, L17

Hernández, J., Hartmann, L., Calvet, N., et al. 2008, ApJ, 686, 1195

Honda, M., Maaskant, K., Okamoto, Y. K., et al. 2012, ApJ, 752, 143

Isella, A., \& Turner, N. 2016, ApJ, submitted (arXiv:1608.05123)

Jang-Condell, H. 2008, ApJ, 679, 797

Jang-Condell, H. 2009, ApJ, 700, 820

Jang-Condell, H., \& Turner, N. J. 2012, ApJ, 749, 153

Jang-Condell, H., \& Turner, N. J. 2013, ApJ, 772, 34

Jin, S., Li, S., Isella, A., Li, H., \& Ji, J. 2016, ApJ, 818, 76

Kanagawa, K. D., Muto, T., Tanaka, H., et al. 2015, ApJL, 806, L15

Kanagawa, K. D., Muto, T., Tanaka, H., et al. 2016a, PASJ, 68, 43

Kanagawa, K. D., Tanaka, H., Muto, T., \& Tanigawa, T. 2016b, MNRAS, submitted (arXiv:1609.02706)

Kim, S.-H., Martin, P. G., \& Hendry, P. D. 1994, ApJ, 422, 164

Kley, W., \& Dirksen, G. 2006, A\&A, 447, 369

Konishi, M., Grady, C. A., Schneider, G., et al. 2016, ApJL, 818, L23

Kraus, A. L., \& Ireland, M. J. 2012, ApJ, 745, 5

Lenzen, R., Hartung, M., Brandner, W., et al. 2003, Proc. SPIE, 4841, 944

Li, H., Finn, J. M., Lovelace, R. V. E., \& Colgate, S. A. 2000, ApJ, 533, 1023

Lin, M.-K., \& Papaloizou, J. C. B. 2010, MNRAS, 405, 1473

Maaskant, K. M., Honda, M., Waters, L. B. F. M., et al. 2013, A\&A, 555, A64

Macintosh, B. A., Graham, J. R., Palmer, D. W., et al. 2008, Proc. SPIE, 7015, 701518

Malik, M., Meru, F., Mayer, L., \& Meyer, M. 2015, ApJ, 802, 56

Momose, M., Morita, A., Fukagawa, M., et al. 2015, PASJ, 67, 83

Mouillet, D., Lagrange, A. M., Augereau, J. C., \& Ménard, F. 2001, A\&A, 372, L61

Muto, T. 2011, ApJ, 739, 10

Muto, T., Suzuki, T. K., \& Inutsuka, S.-i. 2010, ApJ, 724, 448

Osorio, M., Anglada, G., Carrasco-González, C., et al. 2014, ApJL, 791, L36

Panić, O., Hogerheijde, M. R., Wilner, D., \& Qi, C. 2008, A\&A, 491, 219

Perrot, C., Boccaletti, A., Pantin, E., et al. 2016, A\&A, 590, L7

Piétu, V., Dutrey, A., \& Guilloteau, S. 2007, A\&A, 467, 163

Pinilla, P., Birnstiel, T., \& Walsh, C. 2015a, A\&A, 580, A105

Pinilla, P., de Juan Ovelar, M., Ataiee, S., et al. 2015b, A\&A, 573, A9

Qi, C., Ho, P. T. P., Wilner, D. J., et al. 2004, ApJL, 616, L11

Quanz, S. P., Avenhaus, H., Buenzli, E., et al. 2013, ApJL, 766, L2

Raman, A., Lisanti, M., Wilner, D. J., Qi, C., \& Hogerheijde, M. 2006, AJ, 131, 2290

Rapson, V. A., Kastner, J. H., Andrews, S. M., et al. 2015a, ApJL, 803, L10 Rapson, V. A., Kastner, J. H., Millar-Blanchaer, M. A., \& Dong, R. 2015b, ApJL, 815, L26
Reggiani, M., Quanz, S. P., Meyer, M. R., et al. 2014, ApJL, 792, L23

Ribas, Á., Merín, B., Bouy, H., \& Maud, L. T. 2014, A\&A, 561, A54

Rosotti, G. P., Juhasz, A., Booth, R. A., \& Clarke, C. J. 2016, MNRAS, 459, 2790

Ruge, J. P., Wolf, S., Uribe, A. L., \& Klahr, H. H. 2013, A\&A, 549, A97

Sallum, S., Follette, K. B., Eisner, J. A., et al. 2015, Natur, 527, 342

Shakura, N. I., \& Sunyaev, R. A. 1973, A\&A, 24, 337

Simon, M., Dutrey, A., \& Guilloteau, S. 2000, ApJ, 545, 1034

Stolker, T., Dominik, C., Avenhaus, H., et al. 2016a, A\&A, 595, A113

Stolker, T., Dominik, C., Min, M., et al. 2016b, A\&A, 596, A70

Sylvester, R. J., Skinner, C. J., Barlow, M. J., \& Mannings, V. 1996, MNRAS, 279,915

Takami, M., Hasegawa, Y., Muto, T., et al. 2014, ApJ, 795, 71

Tamura, M., Hodapp, K., Takami, H., et al. 2006, Proc. SPIE, 6269, 62690V

Thalmann, C., Grady, C. A., Goto, M., et al. 2010, ApJL, 718, L87

Thalmann, C., Janson, M., Garufi, A., et al. 2016, ApJL, 828, L17

Thalmann, C., Mulders, G. D., Hodapp, K., et al. 2014, A\&A, 566, A51

Thalmann, C., Mulders, G. D., Janson, M., et al. 2015, ApJL, 808, L41

Torres, C. A. O., Quast, G. R., da Silva, L., et al. 2006, A\&A, 460, 695

Torres, C. A. O., Quast, G. R., Melo, C. H. F., \& Sterzik, M. F. 2008, in Handbook of Star Forming Regions, Volume II: The Southern Sky, ed. B. Reipurth (San Francisco, CA: ASP), 757

van Boekel, R., Henning, T., Menu, J., et al. 2016, ApJ, in press (arXiv:1610. 08939)

van den Ancker, M. E., de Winter, D., \& Tjin A Djie, H. R. E. 1998, A\&A, 330,145

van der Marel, N., van Dishoeck, E. F., Bruderer, S., Pérez, L., \& Isella, A. 2015, A\&A, 579, A106

van der Plas, G., Wright, C. M., Ménard, F., et al. 2017, A\&A, 597, A32

van Leeuwen, F. 2007, A\&A, 474, 653

Wagner, K. R., Sitko, M. L., Grady, C. A., et al. 2015, ApJ, 798, 94

Wahhaj, Z., Cieza, L., Koerner, D. W., et al. 2010, ApJ, 724, 835

Walsh, C., Juhász, A., Meeus, G., et al. 2016, ApJ, 831, 200

Weinberger, A. J., Becklin, E. E., Schneider, G., et al. 1999, ApJL, 525, L53

Whitney, B. A., Robitaille, T. P., Bjorkman, J. E., et al. 2013, ApJS, 207, 30

Wichmann, R., Krautter, J., Covino, E., et al. 1997, A\&A, 320, 185

Wolf, S., \& D'Angelo, G. 2005, ApJ, 619, 1114

Yen, H.-W., Liu, H. B., Gu, P.-G., et al. 2016, ApJL, 820, L25

Zhu, Z., Dong, R., Stone, J. M., \& Rafikov, R. R. 2015, ApJ, 813, 88

Zhu, Z., Nelson, R. P., Dong, R., Espaillat, C., \& Hartmann, L. 2012, ApJ, 755,6

Zhu, Z., Nelson, R. P., Hartmann, L., Espaillat, C., \& Calvet, N. 2011, ApJ, 729,47

Zhu, Z., Stone, J. M., \& Rafikov, R. R. 2013, ApJ, 768, 143

Zhu, Z., Stone, J. M., Rafikov, R. R., \& Bai, X.-N. 2014, ApJ, 785, 122 